\title{
Induced Atanassov's interval-valued intuitionistic fuzzy hybrid Choquet integral operators and their application in decision making
}

\author{
Fanyong Meng * \\ School of M anagement, Qingdao Technological U niversity, \\ Qingdao, 266520, Shandong, China \\ Hao Cheng \\ School of M anagement, Qingdao Technological U niversity, \\ Qingdao, 266520, Shandong, China \\ Qiang Zhang \\ School of M anagement and Economics, B eijing Institute of Technology, \\ Beijing, 100081, China
}

Received 27 August 2012

Accepted 26 February 2013

\begin{abstract}
Based on the Choquet integral and the generalized Shapley function, two new induced Atanassov's interval-valued intuitionistic fuzzy hybrid aggregation operators are defined, which are named as the induced generalized Shapley Atanassov's interval-valued intuitionistic fuzzy hybrid Choquet arithmetical averaging (IGS-IVIFHCAA) operator and the induced generalized Shapley Atanassov's interval-valued intuitionistic fuzzy hybrid Choquet geometric mean (IGSIVIFHCGM) operator. These operators do not only globally consider the importance of elements and their ordered positions, but also overall reflect the correlations among them and their ordered positions. Meantime, some important cases are examined, and some desirable properties are studied. Furthermore, if the information about the weighting vectors is incompletely known, the models for the optimal $\lambda$-fuzzy measures on attribute set and ordered set are established, respectively. Moreover, an approach to multi-attribute decision making under Atanassov's interval-valued intuitionistic fuzzy environment is developed. Finally, a numerical example is provided to illustrate the proposed procedure.
\end{abstract}

Keywords: Multi-attribute decision making; Atanassov's interval-valued intuitionistic fuzzy set; Choquet integral; Generalized Shapley function

\section{Introduction}

A wide range of aggregation operators are found in the literature. One common aggregation method is the ordered weighted averaging (OWA) operator [1]. It provides a parameterized family of aggregation operators that include as special cases the maximum, the minimum and the average. Its fundamental aspect is a reordering step in which the input arguments are rearranged in descending order and the weighting vector is merely associated with its ordered position. Since it was proposed in 1988, the OWA operator has been widely used in decision making under uncertainty, including expert systems, neural networks, fuzzy systems and controls [1-14]. Based on the geometric mean, $\mathrm{Xu}$ and Yager [15] developed the ordered weighted geometric (OWG) operator to aggregate the arguments in a similar way as the OWA operator. Yager and $\mathrm{Xu}$ [16] developed the continuous OWG (COWG) operator. Wu et al. [17, 18] defined the induced continuous OWG (ICOWG) operator

* Corresponding author. Tel.: +86-18254298903; Fax: +86-0532-86875851.

E-mail address: mengfanyongtjie@163.com. 
F anyong $M$ eng et al.

and the induced linguistic ordered weighted geometric (ILOWG) operator. Wei [19] developed the induced Atanassov's intuitionistic fuzzy ordered weighted geometric (I-IFOWG) operator and the induced Atanassov's interval-valued intuitionistic fuzzy ordered weighted geometric (I-IIFOWG) operator. Furthermore, $\mathrm{Xu}$ and Chen $[20,21]$ proposed some geometric aggregation operators and some arithmetic aggregation operators on Atanassov's interval-valued intuitionistic fuzzy sets (IVIFSs). Later, some authors found the ordered weighted operators only consider the importance of the elements' ordered positions, but do not give the importance of their own [22]. In 2003, $\mathrm{Xu}$ and $\mathrm{Da}$ [22] proposed the hybrid weighted averaging (HWA) operator, which does not only consider the ordered positions of the arguments, but also give the importance of them. After the pioneering work of Xu and Da [22], many developed forms are proposed [23-27].

All above aggregation operators are based on the assumption that the discussed elements are independent, i.e., they only consider the addition of the importance of individual elements. However, in many practical situations, the elements in a set are usually correlative [28-40]. When there exist inter-dependent or correlative characteristics among elements, it is unreasonable to aggregate the values for elements by using additive measures. Based on the Choquet integral [41], many aggregation operators are proposed, such as the Choquet integral operator on fuzzy sets [30], the Atanassov's intuitionistic fuzzy correlated averaging (IFCA) operator [31, 32], the Atanassov's intuitionistic fuzzy correlated geometric (IFCG) operator [31], the Atanassov's interval-valued intuitionistic fuzzy correlated averaging (IVIFCA) operator [31, 33], the Atanassov's interval-valued intuitionistic fuzzy correlated geometric (IVIFCG) operator [31], the induced Atanassov's intuitionistic fuzzy Choquet ordered averaging (IIFCOA) operator [34], the combined continuous generalized Choquet integral aggregation (CC-GCIA) operator [35] and the induced generalized Atanassov's interval-valued intuitionistic fuzzy Choquet ordered averaging (I-GIVIFCOA) operator [36]. These operators do not only consider the importance of elements or their ordered positions, but also can reflect the correlations among them or their ordered positions. But all existing Choquet integral aggregation operators neither globally consider the importance of elements or their ordered positions nor overall reflect the correlations among them or their ordered positions.

The purpose of this paper is to develop two new induced Atanassov's interval-valued intuitionistic fuzzy hybrid aggregation operators, which do not only globally consider the importance of elements and their ordered positions, but also overall reflect the correlations among them and their ordered positions. It is worth pointing out that most of the existing Atanassov's intuitionistic fuzzy aggregation operators are special cases of our operators. Since the fuzzy measure is defined on the power set, it makes the problem exponentially complex. In order to globally reflect the interaction among elements and simplify the complexity of solving a fuzzy measure, we further introduce the induced generalized $\lambda$-Shapley Atanassov's interval-valued intuitionistic fuzzy hybrid Choquet arithmetical averaging (IG $\lambda \mathrm{S}-I V I F H C A A)$ operator and the induced generalized $\lambda$-Shapley Atanassov's interval- valued intuitionistic fuzzy hybrid Choquet geometric mean (IG $\lambda \mathrm{S}-\mathrm{IVIFHCGM}$ ) operator. Furthermore, the models for the optimal $\lambda$-fuzzy measures on attribute set and ordered set are respectively built. Moreover, an approach to Atanassov's interval-valued intuitionistic fuzzy multi-attribute decision making with incomplete weighting information and correlative conditions is developed.

The rest parts of this paper are organized as follows: In section 2, some conceptions and definitions are reviewed, such as the HWAA and HWGM operators, Atanassov's interval-valued intuitionistic fuzzy sets, fuzzy measure and Choquet integral. In section 3, the IGS-IVIFHCAA and IGS-IVIFHCGM operators are defined. Some important cases and desirable properties are studied. In section 4, the IG $\lambda$ S-IVIFHCAA and IG $\lambda$ S-IVIFHCGM operators are defined. The models for the optimal $\lambda$-fuzzy measures are established, and an approach to multi-attribute decision making under Atanassov's interval-valued intuitionistic fuzzy environment is developed. In section 5, an example is provided to illustrate the developed procedure. The conclusions are made in the last section. 


\section{Preliminaries}

\subsection{The HWAA and HWGM operators}

Based on the weighted arithmetical averaging (WAA) operator and the OWA operator, $\mathrm{Xu}$ and $\mathrm{Da}$ [22] proposed the hybrid weighted averaging (HWA) operator as follows:

Definition 2.1 [22] A HWA operator of dimension $n$ is a mapping HWA: $\mathrm{R}^{\mathrm{n}} \rightarrow \mathrm{R}$ which has an associated weighting vector $W=\left(w_{1}, w_{2}, \ldots, w_{n}\right)^{\top}$ such that $w_{j} \in[0,1]$ and $\sum_{j=1}^{n} w_{j}=1$, denoted by

$$
\operatorname{HWA}_{w, \omega}\left(a_{1}, a_{2}, \ldots, a_{n}\right)=\sum_{j=1}^{n} w_{j} b_{j},
$$

where $b_{j}$ is the jth largest of the weighted arguments $n \omega_{j} a_{j}$ $(j=1,2, \ldots, n), \omega=\left(\omega_{1}, \omega_{2}, \ldots, \omega_{n}\right)^{\top}$ is the weighting vector of $a_{i}(i=1,2, \ldots, n)$ with $\omega_{i}>0$ and $\sum_{i=1}^{n} \omega_{i}=1$, and $n$ is the balancing coefficient.

Based on the weighted geometric mean (WGM) operator and the OWG operator, $\mathrm{Xu}$ and $\mathrm{Da}$ [42] further developed the hybrid weighted geometric mean (HWGM) operator to aggregate the arguments in a similar way as the HWA operator as follows:

Definition 2.2 [42] A HWGM operator of dimension $n$ is a mapping HWGM: $\mathrm{R}^{\mathrm{n}} \rightarrow \mathrm{R}$ which has an associated weighting vector $W=\left(W_{1}, W_{2}, \ldots, W_{n}\right)^{\top}$ such that $w_{j} \in[0,1]$ and $\sum_{j=1}^{n} w_{j}=1$, denoted by

$$
\operatorname{HWGM}_{w, \omega}\left(a_{1}, a_{2}, \ldots, a_{n}\right)=\prod_{j=1}^{n} b_{j}^{w_{j}}
$$

where $b_{j}$ is the $j$ th largest of the weighted arguments $a_{j}{ }^{n \omega_{j}}$ $(j=1,2, \ldots, n), \omega=\left(\omega_{1}, \omega_{2}, \ldots, \omega_{n}\right)^{\top}$ is the weighting vector of $a_{i}(i=1,2, \ldots, n)$ with $\omega_{i}>0$ and $\sum_{i=1}^{n} \omega_{i}=1$, and $n$ is the balancing coefficient.

As Lin and Jiang [23] pointed, the HWA and HWGM operators do not satisfy boundary and idempotent, which are desirable properties for aggregating a finite collection of arguments. Lin and Jiang [23] further proposed the following hybrid weighted arithmetical averaging (HWAA) operator.

Definition 2.3 [23] A HWAA operator of dimension $n$ is a mapping HWAA: $\mathrm{R}^{\mathrm{n}} \rightarrow \mathrm{R}$ which has an associated weighting vector $W=\left(w_{1}, w_{2}, \ldots, w_{n}\right)^{\top}$ on $N=\{1,2, \ldots, n\}$ such that $w_{j} \in[0,1]$ and $\sum_{j=1}^{n} w_{j}=1$, denoted by

$$
\operatorname{HWAA}_{w, \omega}\left(a_{1}, a_{2}, \ldots, a_{n}\right)=\frac{\sum_{j=1}^{n} w_{j} \omega_{(j)} a_{(j)}}{\sum_{j=1}^{n} w_{j} \omega_{(j)}},
$$

where $(\cdot)$ is a permutation on $\mathrm{N}$ such that $\mathrm{a}_{(\mathrm{j})}$ is the $\mathrm{jth}$ largest of $a_{i}$, and $\omega=\left(\omega_{1}, \omega_{2}, \ldots, \omega_{n}\right)^{\top}$ is the weighting vector of $a_{i}$ $(i=1,2, \ldots, n)$ with $\omega_{1}>0$ and $\sum_{i=1}^{n} \omega_{i}=1$.

\subsection{Atanassov's interval-valued intuitionistic fuzzy sets}

By extending Zadeh's fuzzy sets, Atanassov and Gargov [43] introduced the concept of Atanassov's interval-valued intuitionistic fuzzy sets (IVIFSs) as follows:

Definition 2.4 [43] Let $X$ be a no empty finite set. An IVIFS $A$ in $X$ is expressed as

$$
A=\left\{\left\langle x,\left[u_{A}^{-}(x), u_{A}^{+}(x)\right],\left[v_{A}^{-}(x), v_{A}^{+}(x)\right]\right\rangle \mid x \in X\right\},
$$

where $\left[\mathrm{u}_{\mathrm{A}}^{-}(\mathrm{x}), \mathrm{u}_{\mathrm{A}}^{+}(\mathrm{x})\right] \subseteq[0,1]$ and $\left[\mathrm{v}_{\mathrm{A}}^{-}(\mathrm{x}), \mathrm{v}_{\mathrm{A}}^{+}(\mathrm{x})\right] \subseteq[0,1]$ respectively denote the interval-valued degrees of membership and non-membership of element $x \in X$ with the condition $\mathrm{u}_{\mathrm{A}}^{+}(\mathrm{x})+\mathrm{V}_{\mathrm{A}}^{+}(\mathrm{x}) \leq 1$.

If $u_{A}^{-}(x)=u_{A}^{+}(x)$ and $v_{A}^{-}(x)=v_{A}^{+}(x)$ for each $x \in X$, then the IVIFS $A$ degenerates to be an IFS, denoted by $A=$ $\left\{\left\langle x,\left[u_{A}^{-}(x), v_{A}^{-}(x),\right]\right\rangle \mid x \in X\right\}$. Namely, IFS is a special case of IVIFS. Furthermore, if $u_{A}^{-}(x)=1-v_{A}^{-}(x)$, then we get a fuzzy set, expressed by $A=\left\{\left\langle x,\left[u_{A}^{-}(x), 1-u_{A}^{-}(x),\right]\right\rangle \mid x \in X\right\}$.

In order to denote simply, any Atanassov's intervalvalued intuitionistic fuzzy value (IVIFV) $\tilde{\alpha}$ is given as 
F anyong M eng et al.

$\tilde{\alpha}=([a, b],[c, d]) \quad($ see $[20])$, where $[a, b] \subseteq[0,1]$ and $[c, d] \subseteq[0,1]$ respectively denote the interval-valued degrees of membership and non-membership with the condition $b+d \leq 1$. By $\Omega$, we denote the set of all IVIFVs.

For any IVIFV $\tilde{\alpha}=([a, b],[c, \mathrm{~d}]), \mathrm{Xu}[44]$ introduced the score function $S(\tilde{\alpha})=0.5(a-c+b-d)$ to get the score of $\tilde{\alpha}$, and defined the accuracy degree $\mathrm{H}(\tilde{\alpha})=0.5(a+c+b+d)$ to evaluate the accuracy degree of $\tilde{\alpha}$. Xu [44] further gave the following order relationship between any two IVIFVs $\tilde{\alpha}_{1}$ and $\tilde{\alpha}_{2}$.

$$
\begin{aligned}
& \text { If } S\left(\tilde{\alpha}_{1}\right)<S\left(\tilde{\alpha}_{2}\right) \text {, then } \tilde{\alpha}_{1}<\tilde{\alpha}_{2} . \\
& \text { If } S\left(\tilde{\alpha}_{1}\right)=S\left(\tilde{\alpha}_{2}\right) \text {, then }\left\{\begin{array}{l}
\mathrm{H}\left(\tilde{\alpha}_{1}\right)=H\left(\tilde{\alpha}_{2}\right) \Rightarrow \tilde{\alpha}_{1}=\tilde{\alpha}_{2} \\
\mathrm{H}\left(\tilde{\alpha}_{1}\right)<H\left(\tilde{\alpha}_{2}\right) \Rightarrow \tilde{\alpha}_{1}<\tilde{\alpha}_{2}
\end{array} .\right.
\end{aligned}
$$

Definition $2.5[20,21]$ Let $\tilde{\alpha}=([a, b],[c, d])$ and $\tilde{\gamma}=([e, f],[\mathrm{g}, \mathrm{h}])$ be any two IVIFVs, then some operations of $\tilde{\alpha}$ and $\tilde{\gamma}$ are defined by

(1) $\tilde{\alpha} \oplus \tilde{\gamma}=([a+e-\mathrm{ae}, \mathrm{b}+\mathrm{f}-\mathrm{bf}],[\mathrm{cg}, \mathrm{dh}])$,

(2) $\tilde{\alpha} \otimes \tilde{\gamma}=([a e, b f],[\mathrm{c}+\mathrm{g}-\mathrm{cg}, \mathrm{d}+\mathrm{h}-\mathrm{dh}])$,

(3) $r \tilde{\alpha}=\left(\left[1-(1-a)^{r}, 1-(1-b)^{r}\right],\left[c^{x}, d_{1}\right]\right) r>0$,

(4) $\tilde{\alpha}^{r}=\left(\left[a^{r}, b^{r}\right],\left[1-(1-c)^{r}, 1-(1-\mathrm{d})^{r}\right]\right) r>0$.

Proposition 2.1 [46] Let $\tilde{\alpha}=([a, b],[\mathrm{c}, \mathrm{d}])$ and $\tilde{\gamma}=([e, f],[\mathrm{g}, \mathrm{h}])$ be two IVIFVs, then

(1) $\tilde{\alpha} \oplus \tilde{\gamma}=\tilde{\gamma} \oplus \tilde{\alpha}$,

(2) $\tilde{\alpha} \otimes \tilde{\gamma}=\tilde{\gamma} \otimes \tilde{\alpha}$,

(3) $\mathrm{r}(\tilde{\tilde{\alpha}} \oplus \bar{\gamma})=r \tilde{\alpha} \oplus r \tilde{\gamma} \quad r>0$,

(4) $(\tilde{\alpha} \otimes \tilde{\gamma})^{r}=\tilde{\tilde{\alpha}}^{r} \otimes \tilde{\gamma}^{r} \quad r>0$,

(5) $\left(\mathrm{r}_{1}+\mathrm{r}_{2}\right) \bar{\alpha}=r_{1} \bar{\alpha} \oplus r_{2} \bar{\alpha} \quad r_{1}, r_{2}>0$.

(6) $\tilde{\alpha}^{r_{1}+r_{2}}=\tilde{\alpha}^{r_{1}} \otimes \tilde{\alpha}^{r_{2}} \quad r_{1}, r_{2}>0$.

\subsection{Fuzzy measure and Choquet integral}

As an effective tool to measure the importance of elements and the interaction among them, fuzzy measure (see [47]) has been deeply studied by many researchers
[47-50], and successfully used in many different fields, especially in game theory and decision making [28-40].

Let $N=\{1,2, \ldots, n\}$ be a finite set, and $P(N)$ be the power set of $\mathrm{N}$, i.e., the set of all subsets of $\mathrm{N}$. We will often omit braces for singletons, e.g., by writing $\mathrm{N} \backslash \mathrm{i}, \mathrm{T}, \mathrm{N} \backslash \mathrm{S}$ instead of $N \backslash\{i\},\{T\}, N \backslash\{S\}$, respectively. Moreover, the cardinality of any subset $S \in P(N)$ will be denoted by the corresponding lower case $\mathrm{s}$.

Definition 2.6 [47] A fuzzy measure on $\mathrm{N}$ is a set function $\mu$ : $\mathrm{P}(\mathrm{N}) \rightarrow[0,1]$ satisfying

(1) $\mu(\varnothing)=0, \mu(\mathrm{N})=1$,

(2) If $\mathrm{A}, \mathrm{B} \in \mathrm{P}(\mathrm{N})$ with $\mathrm{A} \subseteq \mathrm{B}$, then $\mu(\mathrm{A}) \leq \mu(\mathrm{B})$.

Fuzzy integrals, as important aggregation operators for uncertain information, have been studied by many researchers. One of the most important fuzzy integrals is the Choquet integral. In 1997, Grabisch [48] gave the following concept of the Choquet integral on discrete sets.

Definition 2.7 [48] Let $f$ be a positive real-valued function on $\mathrm{X}$, and $\mu$ be a fuzzy measure on $\mathrm{N}$. The discrete Choquet integral of $f$ w.r.t. $\mu$ is defined by

$C_{\mu}\left(f\left(x_{(1)}\right), f\left(x_{(2)}\right), \ldots, f\left(x_{(n)}\right)\right)=\sum_{i=1}^{n} f\left(x_{(i)}\right)\left(\mu\left(A_{(i)}\right)-\mu\left(A_{(i+1)}\right)\right)$,

where (.) indicates a permutation on $N$ such that $f\left(x_{(1)}\right) \leq$ $\mathrm{f}\left(\mathrm{x}_{(2)}\right) \leq \ldots \leq f\left(x_{(n)}\right) \quad, \quad$ and $\quad \mathrm{A}_{(\mathrm{i})}=\left\{\mathrm{x}_{(\mathrm{i})}, \ldots, \mathrm{x}_{(\mathrm{n})}\right\} \quad$ with $A_{(n+1)}=\varnothing$.

\section{New Atanassov's interval-valued intuitionistic fuzzy aggregation operators}

In this section, we shall define the generalized Shapley Atanassov's interval-valued intuitionistic fuzzy hybrid Choquet arithmetical averaging (GS-IVIFHCAA) operator and the generalized Shapley Atanassov's interval-valued intuitionistic fuzzy hybrid Choquet geometric mean (GS-IVIFHCGM) operator. Some important cases and desirable properties are studied.

First, we introduce the generalized Shapley function 
proposed by Marichal [51] for game theory as follows:

$\Phi_{\mathrm{S}}(\mu, \mathrm{N})=\sum_{\mathrm{T} \subseteq \mathrm{N} \backslash \mathrm{S}} \frac{(\mathrm{n}-\mathrm{S}-\mathrm{t}) ! \mathrm{t} !}{(\mathrm{n}-\mathrm{S}+1) !}(\mu(\mathrm{S} \cup T)-\mu(\bar{T})) \forall \mathrm{S} \subseteq \mathrm{N}$,

where $\mu$ is a fuzzy measure on $\mathrm{N}$.

When $s=1$, then Eq.(2) degenerates to be the Shapley function [52]

$$
\varphi_{\mathrm{i}}(\mu, \mathrm{N})=\sum_{\mathrm{S} \subseteq \mathrm{N} \backslash \mathrm{i}} \frac{(\mathrm{n}-\mathrm{s}-1) ! \mathrm{s} !}{\mathrm{n} !}(\mu(\mathrm{S} \cup i)-\mu(S)) \quad \forall \mathrm{i} \in \mathrm{N},
$$

From Eq.(2), we know the generalized Shapley function is an expect value of the globally marginal contributions between the coalition $S$ and every coalition in $N \backslash S$. While Eq.(3) gives an expect value of the overall marginal contributions between the element $i$ and every coalition in $\mathrm{N} \backslash$ i. From Eq.(2), it is not difficult to get the following theorem.

Theorem 3.1 Let $\mu: \mathrm{P}(\mathrm{N}) \rightarrow[0,1]$ be a fuzzy measure on $\mathrm{N}$, then $\Phi$ given as Eq.(2) is also a fuzzy measure.

From Definition 2.6 and Eq.(3), one can easily get the following two properties.

Property 3.1 Let $\mu: \mathrm{P}(\mathrm{N}) \rightarrow[0,1]$ be a fuzzy measure on the finite set $\mathrm{N}$, then $\varphi_{i}(\mu, \mathrm{N}) \geq 0$ for each element $i \in \mathrm{N}$.

Property 3.2 Let $\mu: \mathrm{P}(\mathrm{N}) \rightarrow[0,1]$ be a fuzzy measure on the finite set $\mathrm{N}$, then $\sum_{\mathrm{i}=1}^{\mathrm{n}} \varphi_{\mathrm{i}}(\mu, \mathrm{N})=\mu(\mathrm{N})=1$.

From Properties 3.1 and 3.2, it is not difficult to know that $\left\{\varphi_{i}(\mu, N)\right\}_{i \in N}$ is a weighting vector.

\subsection{The IGS-IVIFHCAA and IGS-IVIFHCGM operators}

Considering a set of two tuples $<u_{1}, \tilde{\alpha}_{1}>,<u_{2}, \tilde{\alpha}_{2}>, \ldots,<u_{n}, \tilde{\alpha}_{n}>$, where $u_{i}(i=1,2, \ldots, n)$ indicates the order-inducing variables. Let $\mu$ and $v$ be a fuzzy measure on ordered set $N=\{1,2, \ldots, n\}$ and IVIFS $\tilde{\vartheta}=\left\{\tilde{\alpha}_{i}\right\}_{i \in N}$, respectively. $\varphi_{\tilde{a}_{i}}(\mathcal{V}, \tilde{\vartheta})$ is the Shapley function w.r.t. the fuzzy measure $v$ on $\bar{\vartheta}=\left\{\tilde{\alpha}_{i}\right\}_{i \in N}$ for $\tilde{\alpha}_{i}(\mathrm{i}=1$, $2, \ldots, n)$, and $\Phi_{A(j)}(\mu, N)$ is the generalized Shapley value w.r.t. the fuzzy measure $\mu$ on ordered set $N=\{1,2, \ldots, n\}$ for $A_{(j)}=\{j, \ldots, n\}$ with $A_{(n+1)}=\varnothing$, where $(\cdot)$ is a permutation on $u_{i}(i=1,2, \ldots, n)$ such that $u_{(j)}$ being the $j$ th largest of $u_{i}$ $(i=1,2, \ldots, n)$.

Example 3.1 Consider the following collection of two tuples:

$$
\begin{aligned}
& <\mathrm{u}_{1}, \tilde{\alpha}_{1}>=<0.5,([0.3,0.6],[0.2,0.4])>, \\
& <\mathrm{u}_{2}, \tilde{\alpha}_{2}>=<0.3,([0.2,0.5],[0.3,0.4])>, \\
& <\mathrm{u}_{3}, \tilde{\alpha}_{3}>=<0.6,([0.5,0.7],[0.1,0.3])>.
\end{aligned}
$$

Since $\mathrm{u}_{3}>\mathrm{u}_{1}>\mathrm{u}_{2}$, we get $\tilde{\alpha}_{(1)}=\tilde{\alpha}_{3}, \tilde{\alpha}_{(2)}=\tilde{\alpha}_{1}$ and $\tilde{\alpha}_{(3)}=\tilde{\alpha}_{2}$. Assume that the fuzzy measure $\mu$ on IVIFS $\tilde{\vartheta}=\left\{\tilde{\alpha}_{i}\right\}_{i \in N}(\mathrm{~N}$ $=\{1,2,3\})$ is defined by

$$
\begin{aligned}
& \mu(\varnothing)=0, \mu\left(\overline{\tilde{\alpha}}_{1}\right)=0.3, \mu\left(\overline{\tilde{\alpha}}_{2}\right)=\mu\left(\overline{\tilde{\alpha}}_{3}\right)=0.2, \mu\left(\tilde{\alpha}_{1}, \tilde{\alpha}_{2}\right)= \\
& \mu\left(\tilde{\alpha}_{2}, \tilde{\alpha}_{3}\right)=0.7, \mu\left(\tilde{\alpha}_{1}, \bar{\alpha}_{3}\right)=0.6, \mu\left(\tilde{\alpha}_{1}, \tilde{\alpha}_{2}, \tilde{\alpha}_{3}\right)=1,
\end{aligned}
$$

and the fuzzy measure $\mu$ on ordered set $\mathrm{N}=\{1,2,3\}$ is given as

$$
\begin{aligned}
& \mu(\varnothing)=0, \mu(1)=\mu(2)=0.2, \mu(3)=0.4, \mu(1,2)=0.6, \\
& \mu(1,3)=\mu(2,3)=0.7, \mu(1,2,3)=1 .
\end{aligned}
$$

Then, we get the Shapley values of IVIFVs $\left\{\tilde{\alpha}_{i}\right\}_{i \in N}$ as follows:

$$
\varphi_{\tilde{a}_{1}}(\grave{v}, \bar{\vartheta})=\varphi_{\tilde{a}_{2}}(v, \bar{\vartheta})=0.28 \varphi_{\bar{a}_{3}}(v, \bar{\vartheta})=0.43
$$

and the generalized Shapley values of the ordered positions are obtained by

$$
\Phi_{\mathrm{N}}(\mu, \mathrm{N})=1, \Phi_{\{1,2\}}(\mu, \mathrm{N})=0.6, \Phi_{2}(\mu, \mathrm{N})=0.28 \text {. }
$$

In the following, we present two hybrid aggregation operators, which can deal with the situations as given in Example 3.1.

Definition 3.1 An IGS-IVIFHCAA operator of dimension $n$ is a mapping IGS-IVIFHCAA: $\Omega^{n} \rightarrow \Omega$ defined on the set of second arguments of two tuples $\left.\left\langle\mathrm{u}_{1}, \tilde{\alpha}_{1}\right\rangle,<u_{2}, \tilde{\alpha}_{2}\right\rangle, \ldots$, $<\mathrm{u}_{\mathrm{n}}, \tilde{\alpha}_{n}>$ with a set of order-inducing variables $\mathrm{u}_{\mathrm{i}}(\mathrm{i}=1,2, \ldots$, denoted by

$\operatorname{IGS}_{\operatorname{IVIFHCAA}}\left(<\mathrm{u}_{1}, \tilde{\alpha}_{1}>,<u_{2}, \tilde{\alpha}_{2}>, \ldots,<u_{n}, \tilde{\alpha}_{n}>\right)$ 
F anyong M eng et al.

$$
=\frac{\bigoplus_{\mathrm{j}=1}^{\mathrm{n}}\left(\Phi_{\mathrm{A}_{(\mathrm{j})}}(\mu, \mathrm{N})-\Phi_{\mathrm{A}_{(j+1)}}(\mu, \mathrm{N})\right) \varphi_{\tilde{a}_{(j)}}(\nu, \tilde{\vartheta}) \tilde{\alpha}_{(j)}}{\sum_{\mathrm{j}=1}^{\mathrm{n}}\left(\Phi_{\mathrm{A}_{(\mathrm{j})}}(\mu, \mathrm{N})-\Phi_{\mathrm{A}_{(j+1)}}(\mu, \mathrm{N})\right) \varphi_{\tilde{a}_{(j)}}(\dot{v}, \tilde{\vartheta})},
$$

where the notations as given above.

Theorem 3.2 Let $\tilde{\alpha}_{i}=\left(\left[a_{i}, b_{i}\right],\left[\mathrm{c}_{\mathrm{i}}, \mathrm{d}_{\mathrm{i}}\right]\right)(\mathrm{i}=1,2, \ldots, \mathrm{n})$ be a collection of IVIFVs in $\Omega$, then their aggregated value by using the IGS-IVIFHCAA operator is also an IVIFV, denoted by

$$
\begin{aligned}
& \operatorname{IGS}-I V I F H C A A_{\Phi, \varphi}\left(<\mathrm{u}_{1}, \tilde{\alpha}_{1}>,<u_{2}, \tilde{\alpha}_{2}>, \ldots,<u_{n}, \tilde{\alpha}_{n}>\right) \\
& =\left(\left[1-\prod_{j=1}^{n}\left(1-a_{(j)}\right)^{\frac{\left(\Phi_{A_{(j)}}(\mu, \mathrm{N})-\Phi_{A_{(j+1)}}(\mu, \mathrm{N})\right) \varphi_{a_{(j)}}(v, \tilde{\vartheta})}{\sum_{j=1}^{n}\left(\Phi_{A_{(j)}}(\mu, \mathrm{N})-\Phi_{A_{(j+1)}}(\mu, \mathrm{N})\right) \varphi_{\bar{a}_{(j)}}(v, \tilde{\vartheta})}},\right.\right. \\
& \left.1-\prod_{j=1}^{n}\left(1-b_{(j)}\right)^{\frac{\left(\Phi_{A_{(j)}}(\mu, N)-\Phi_{A_{(j+1)}}(\mu, \mathrm{N})\right) \varphi_{\hat{a}_{(j)}}(v, \tilde{\vartheta})}{\sum_{j=1}^{n}\left(\Phi_{A_{(j)}}(\mu, \mathrm{N})-\Phi_{A_{(j+1)}}(\mu, \mathrm{N})\right) \varphi_{\tilde{a}_{(j)}}(v, \tilde{\vartheta})}}\right],
\end{aligned}
$$

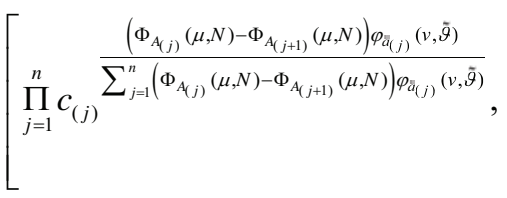

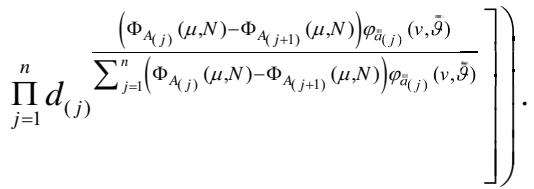

Proof. The first result follows quickly from Definition 2.4. Below we prove Eq.(5) by using mathematical induction on n.

(i) When $n=2$, from Theorem 3.1 and Properties 3.1 and 3.2 , we get

$$
\frac{\left(\Phi_{\mathrm{A}_{(\mathrm{j})}}(\mu, \mathrm{N})-\Phi_{\mathrm{A}_{(j+1)}}(\mu, \mathrm{N})\right) \varphi_{\tilde{a}_{(j)}}(\nu, \tilde{\vartheta})}{\sum_{\mathrm{j}=1}^{\mathrm{n}}\left(\Phi_{\mathrm{A}_{(\mathrm{j})}}(\mu, \mathrm{N})-\Phi_{\mathrm{A}_{(j+1)}}(\mu, \mathrm{N})\right) \varphi_{\tilde{a}_{(j)}}(\mathcal{V}, \tilde{\vartheta})} \geq 0
$$

By Definition 2.5, we have

$$
\frac{\left(\Phi_{\mathrm{A}_{(\mathrm{j})}}(\mu, \mathrm{N})-\Phi_{\mathrm{A}_{(\mathrm{j}+1)}}(\mu, \mathrm{N})\right) \varphi_{\tilde{a}_{(j)}}(\hat{v}, \tilde{\vartheta})}{\sum_{\mathrm{j}=1}^{2}\left(\Phi_{\mathrm{A}_{(\mathrm{j})}}(\mu, \mathrm{N})-\Phi_{\mathrm{A}_{(j+1)}}(\mu, \mathrm{N})\right) \varphi_{\tilde{a}_{(j)}}(\mathfrak{v}, \tilde{\vartheta})} \tilde{\alpha}_{(j)}
$$

$$
=\left(\left[1-\left(1-\mathrm{a}_{(\mathrm{j})}\right)^{\frac{\left(\Phi_{\left.A_{(\mathrm{j})}\right)}(\mu, \mathrm{N})-\Phi_{A_{(j+1)}}(\mu, \mathrm{N})\right) \varphi_{\bar{a}_{(j)}}(v, \tilde{\vartheta})}{\sum_{\mathrm{j}=1}^{2}\left(\Phi_{A_{(j)}}(\mu, \mathrm{N})-\Phi_{A_{(j+1)}}(\mu, \mathrm{N})\right) \varphi_{\bar{a}_{(j)}}(v, \tilde{\vartheta})}},\right.\right.
$$$$
\left.1-\left(1-\mathrm{b}_{(\mathrm{j})}\right)^{\frac{\left(\Phi_{A_{(j)}}(\mu, \mathrm{N})-\Phi_{A_{(j+1)}}(\mu, \mathrm{N})\right) \varphi_{\bar{a}_{(j)}}(v, \bar{g})}{\sum_{j=1}^{2}\left(\Phi_{A_{(j)}}(\mu, \mathrm{N})-\Phi_{A_{(j+1)}}(\mu, \mathrm{N})\right) \varphi_{\bar{a}_{(j)}}(v, \tilde{g})}}\right],
$$

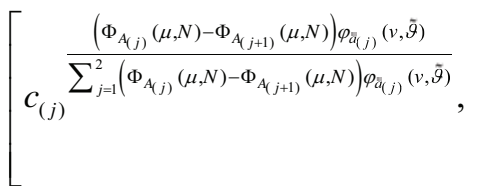$$
\left.\left.\mathrm{d}_{(\mathrm{j})} \frac{\left(\Phi_{\mathrm{A}_{(\mathrm{j})}}(\mu, \mathrm{N})-\Phi_{A_{(\mathrm{j}+1)}}(\mu, \mathrm{N})\right) \varphi_{\bar{a}_{(j)}}(v, \bar{q})}{\sum_{\mathrm{j}=1}^{2}\left(\Phi_{A_{(\mathrm{j})}}(\mu, \mathrm{N})-\Phi_{A_{(j+1)}}(\mu, \mathrm{N})\right) \varphi_{\bar{a}_{(j)}}(v, \tilde{q})}\right]\right)
$$

for $\mathrm{j}=1,2$.

Thus,

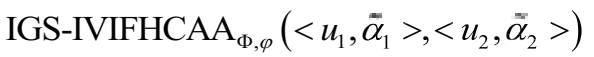

$$
\begin{aligned}
& =\frac{\left(\Phi_{\mathrm{A}_{(1)}}(\mu, \mathrm{N})-\Phi_{\mathrm{A}_{(2)}}(\mu, \mathrm{N})\right) \varphi_{\tilde{a}_{(1)}}(\nu, \tilde{\vartheta}) \tilde{\alpha}_{(1)}}{\sum_{\mathrm{j}=1}^{2}\left(\Phi_{\mathrm{A}_{(\mathrm{j})}}(\mu, \mathrm{N})-\Phi_{\mathrm{A}_{(\mathrm{j}+1)}}(\mu, \mathrm{N})\right) \varphi_{\tilde{a}_{(j)}}(\nu, \tilde{\vartheta})} \oplus \\
& \frac{\left(\Phi_{\mathrm{A}_{(2)}}(\mu, \mathrm{N})-\Phi_{\mathrm{A}_{(3)}}(\mu, \mathrm{N})\right) \varphi_{\tilde{a}_{(2)}}(\nu, \tilde{\vartheta}) \tilde{\alpha}_{(2)}}{\sum_{\mathrm{j}=1}^{2}\left(\Phi_{\mathrm{A}_{(\mathrm{j})}}(\mu, \mathrm{N})-\Phi_{\mathrm{A}_{(\mathrm{j}+1)}}(\mu, \mathrm{N})\right) \varphi_{\tilde{a}_{(j)}}(\nu, \tilde{\vartheta})} \\
& =\left(\left[1-\left(1-a_{(1)}\right)^{\frac{\left(\Phi_{A_{(1)}}(\mu, \mathrm{N})-\Phi_{A_{(2)}}(\mu, \mathrm{N})\right) \varphi_{\bar{a}_{(1)}}(v, \tilde{g})}{\sum_{j=1}^{2}\left(\Phi_{A_{(j)}}(\mu, \mathrm{N})-\Phi_{A_{(j+1)}}(\mu, \mathrm{N})\right) \varphi_{\tilde{a}_{(j)}}(v, \tilde{g})}} \times\right.\right. \\
& \left(1-a_{(2)}\right)^{\frac{\left(\Phi_{A_{(2)}}(\mu, \mathrm{N})-\Phi_{A_{(3)}}(\mu, \mathrm{N})\right) \varphi_{\hat{a}_{(2)}}(\nu, \tilde{\vartheta})}{\sum_{j=1}^{2}\left(\Phi_{A_{(j)}}(\mu, \mathrm{N})-\Phi_{A_{(j+1)}}(\mu, \mathrm{N})\right) \varphi_{\hat{a}_{(j)}}(v, \tilde{\vartheta})}}, \\
& 1-\left(1-\mathrm{b}_{(1)}\right)^{\frac{\left(\Phi_{A_{(1)}}(\mu, \mathrm{N})-\Phi_{A_{(2)}}(\mu, \mathrm{N})\right) \varphi_{a_{(1)}}(v, \grave{q})}{\sum_{j=1}^{2}\left(\Phi_{A_{(j)}}(\mu, \mathrm{N})-\Phi_{A_{(j+1)}}(\mu, \mathrm{N})\right) \varphi_{\bar{a}_{(j)}}(v, \tilde{\vartheta})}} \times \\
& \left.\left(1-\mathrm{b}_{(2)}\right)^{\frac{\left(\Phi_{A_{(2)}}(\mu, \mathrm{N})-\Phi_{A_{(3)}}(\mu, \mathrm{N})\right) \varphi_{\bar{a}(2)}(v, \tilde{\vartheta})}{\sum_{j=1}^{2}\left(\Phi_{A_{(j)}}(\mu, \mathrm{N})-\Phi_{A_{(j+1)}}(\mu, \mathrm{N})\right) \varphi_{\overline{a_{(j)}}}(v, \tilde{\vartheta})}}\right],
\end{aligned}
$$


Interval-valued intuitionistic fuzzy operators

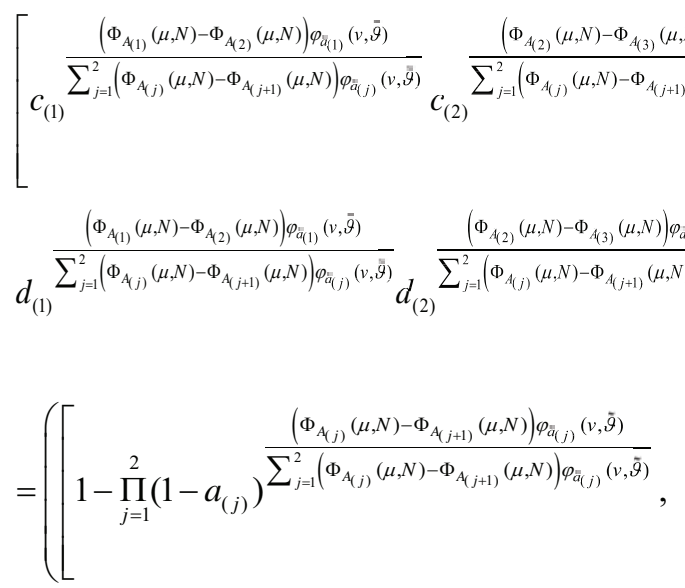

$$
\begin{aligned}
& \left.1-\prod_{\mathrm{j}=1}^{2}\left(1-\mathrm{b}_{(\mathrm{j})}\right)^{\frac{\left(\Phi_{\left.A_{(\mathrm{j})}\right)}(\mu, \mathrm{N})-\Phi_{A_{(\mathrm{j}+1)}}(\mu, \mathrm{N})\right) \varphi_{\tilde{a}_{(j)}}(v, \bar{\vartheta})}{\sum_{j=1}^{2}\left(\Phi_{A_{(j)}}(\mu, \mathrm{N})-\Phi_{A_{(j+1)}}(\mu, \mathrm{N})\right) \varphi_{\bar{a}_{(j)}}(v, \tilde{\vartheta})}}\right],
\end{aligned}
$$

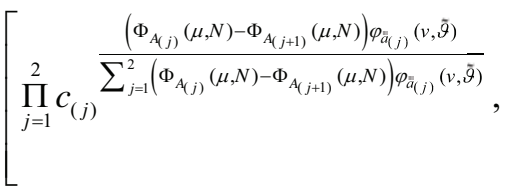

$$
\begin{aligned}
& \left.\left.\prod_{j=1}^{2} d_{(j)} \frac{\left(\Phi_{A_{(j)}}(\mu, \mathrm{N})-\Phi_{A_{(j+1)}}(\mu, N)\right) \varphi_{\hat{a}_{(j)}}(v, \bar{g})}{\sum_{j=1}^{2}\left(\Phi_{A_{(j)}}(\mu, N)-\Phi_{A_{(j+1)}}(\mu, N)\right) \varphi_{a_{(j)}}(v, \tilde{g})}\right]\right) .
\end{aligned}
$$

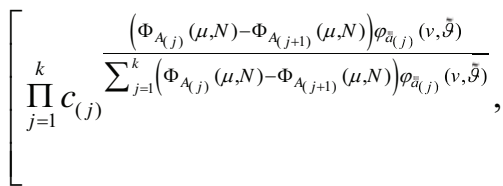

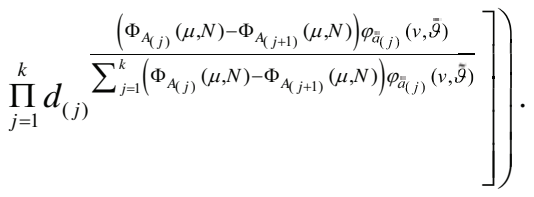

When $n=k+1$, from Proposition 3.1, we get $\operatorname{IGS}$ IVIFHCAA ${ }_{\Phi, \varphi}\left(<u_{1}, \tilde{\alpha}_{1}>,<u_{2}, \tilde{\alpha}_{2}>, \ldots,<u_{k+1}, \tilde{\alpha}_{k+1}>\right)$

$$
\begin{aligned}
& =\left(\left[1-\prod_{j=1}^{k}\left(1-a_{(j)}\right)^{\frac{\left(\Phi_{A_{(j)}}(\mu, N)-\Phi_{A_{(j+1)}}(\mu, N)\right) \varphi_{a_{(j)}}(v, \check{g})}{\sum_{j=1}^{k+1}\left(\Phi_{A_{(j)}}(\mu, N)-\Phi_{A_{(j+1)}}(\mu, N)\right) \varphi_{a_{(j)}}(v, \tilde{\vartheta})}},\right.\right. \\
& \left.1-\prod_{j=1}^{k}\left(1-b_{(j)}\right)^{\frac{\left(\Phi_{A_{(j)}}(\mu, N)-\Phi_{A_{(j+1)}}(\mu, N)\right) \varphi_{\bar{a}_{(j)}}(v, \check{g})}{\sum_{j=1}^{k+1}\left(\Phi_{A_{(j)}}(\mu, N)-\Phi_{A_{(j+1)}}(\mu, \mathrm{N})\right) \varphi_{\tilde{a}_{(j)}}(v, \tilde{g})}}\right],
\end{aligned}
$$

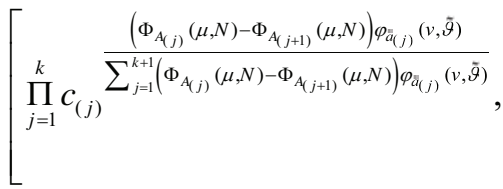

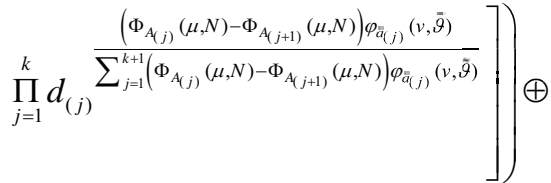

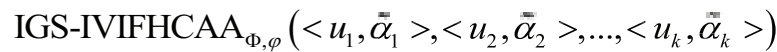

$$
\begin{aligned}
& \left(\left[1-\left(1-a_{(\mathrm{k}+1)}\right)^{\frac{\left(\Phi_{A_{(K+1)}}(\mu, \mathrm{N})-\Phi_{A_{(k+2)}}(\mu, \mathrm{N})\right) \varphi_{\bar{a}_{(k+1)}}(l, \tilde{g})}{\sum_{j=1}^{k+1}\left(\Phi_{\left.A_{(j)}\right)}(\mu, \mathrm{N})-\Phi_{A_{(j+1)}}(\mu, \mathrm{N})\right) \varphi_{\bar{a}_{(j)}}(v, \tilde{\vartheta})}},\right.\right.
\end{aligned}
$$

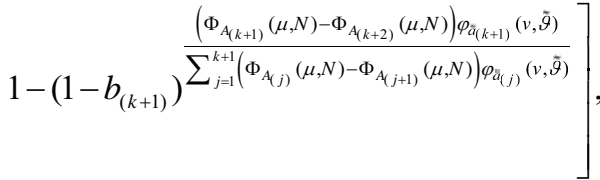


F anyong $M$ eng et al.

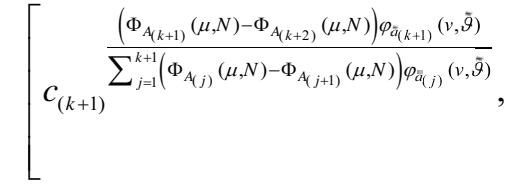

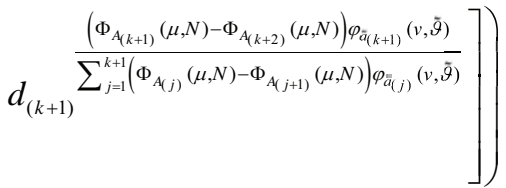

$$
\begin{aligned}
& =\left(\left[1-\prod_{j=1}^{k+1}\left(1-a_{(j)}\right)^{\frac{\left(\Phi_{A_{(j)}}(\mu, \mathrm{N})-\Phi_{A_{(j+1)}}(\mu, \mathrm{N})\right) \varphi_{\tilde{a}_{(j)}}(v, \tilde{q})}{\sum_{j=1}^{k+1}\left(\Phi_{A_{(j)}}(\mu, N)-\Phi_{A_{(j+1)}}(\mu, \mathrm{N})\right) \varphi_{a_{(j)}}(v, \tilde{q})}},\right.\right. \\
& \left.1-\prod_{j=1}^{k+1}\left(1-b_{(j)}\right)^{\frac{\left(\Phi_{A_{(j)}}(\mu, N)-\Phi_{A_{(j+1)}}(\mu, \mathrm{N})\right) \varphi_{\tilde{a}_{(j)}}(v, \tilde{\vartheta})}{\sum_{j=1}^{k+1}\left(\Phi_{A_{(j)}}(\mu, \mathrm{N})-\Phi_{A_{(j+1)}}(\mu, \mathrm{N})\right) \varphi_{\tilde{a}_{(j)}}(v, \tilde{\vartheta})}}\right],
\end{aligned}
$$

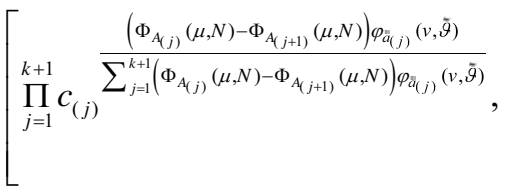

$$
\begin{aligned}
& \left.\left.\prod_{j=1}^{k+1} d_{(j)} \frac{\left(\Phi_{A_{(j)}}(\mu, \mathrm{N})-\Phi_{A_{(j+1)}}(\mu, N)\right) \varphi_{\bar{a}_{(j)}}(v, \tilde{q})}{\sum_{j=1}^{k+1}\left(\Phi_{A_{(j)}}(\mu, \mathrm{N})-\Phi_{A_{(j+1)}}(\mu, \mathrm{N})\right) \varphi_{a_{(j)}}(v, \tilde{g})}\right]\right) .
\end{aligned}
$$

That is, for $n=k+1$, Eq.(5) still holds, and the result is obtained.

Remark 3.1 If $\mu$ and $v$ are both additive, then the IGS-IVIFHCAA operator reduces to be the induced Atanassov's interval-valued intuitionistic fuzzy hybrid weighted arithmetical averaging (I- IVIFHWAA) operator

$$
\begin{aligned}
& \text { I-IVIFHWAA }_{\mathrm{w}, \omega}\left(<\mathrm{u}_{1}, \tilde{\alpha}_{1}>,<u_{2}, \tilde{\alpha}_{2}>, \ldots,<u_{n}, \tilde{\alpha}_{n}>\right) \\
& =\frac{\bigoplus_{\mathrm{j}=1}^{n} \mathrm{w}_{\mathrm{j}} \omega_{(\mathrm{j})} \tilde{\alpha}_{(j)}}{\sum_{\mathrm{j}=1}^{\mathrm{n}} \mathrm{w}_{\mathrm{j}} \omega_{(\mathrm{j})}},
\end{aligned}
$$

where $\mu(\mathrm{i})=\mathrm{w}_{\mathrm{i}}$ and $\mathrm{v}\left(\tilde{\alpha}_{i}\right)=\omega_{i}(\mathrm{i}=1,2, \ldots, \mathrm{n})$.

Furthermore, when $\mathrm{u}_{\mathrm{i}}=\tilde{\alpha}_{i}$ and each IVIFV $\tilde{\alpha}_{i}(\mathrm{i}=1,2, \ldots, \mathrm{n})$ degenerates to be a real number, then IGS-IVIFHCAA operator reduces to be the HWAA operator defined by Lin and Jiang [23].

Remark 3.2 When $u_{i}=\varphi_{\tilde{a}_{i}}(\dot{v}, \tilde{\vartheta}) \tilde{\alpha}_{i}(i=1,2, \ldots, n)$, the IGSIVIFHCAA operator reduces to be the generalized Shapley Atanassov's interval-valued intuitionistic fuzzy hybrid Choquet arithmetical averaging (GS-IVIFHCAA) operator

$$
\begin{aligned}
& \text { GS-IVIFHCAA }{ }_{\Phi, \varphi}\left(\ddot{\alpha}_{1}, \tilde{\alpha}_{2}, \ldots, \tilde{\alpha}_{n}\right) \\
& =\frac{\bigoplus_{j=1}^{n}\left(\Phi_{A_{(j)}}(\mu, \mathrm{N})-\Phi_{A_{(j+1)}}(\mu, \mathrm{N})\right) \varphi_{\tilde{a}_{(j)}}(v, \tilde{\vartheta}) \tilde{\alpha}_{(j)}}{\sum_{j=1}^{n}\left(\Phi_{A_{(j)}}(\mu, \mathrm{N})-\Phi_{A_{(j+1)}}(\mu, N)\right) \varphi_{\tilde{a}_{(j)}}(v, \tilde{\vartheta})},
\end{aligned}
$$
where $\varphi_{\bar{a}_{(j)}}(v, \tilde{\vartheta}) \tilde{\alpha}_{(j)}$ is the jth largest of $\varphi_{\bar{a}_{i}}(v, \tilde{\vartheta}) \tilde{\alpha}_{i}(i=1,2, \ldots$, n).

Remark 3.3 If each IVIFV $\tilde{\alpha}_{i}=\left(\left[a_{i}, b_{i}\right],\left[c_{\mathrm{i}}, \mathrm{d}_{\mathrm{i}}\right]\right)(\mathrm{i}=1,2, \ldots, \mathrm{n})$ degenerates to be an Atanassov's intuitionistic fuzzy value, namely, $\tilde{\alpha}_{i}=\left(a_{i}, c_{i}\right)(\mathrm{i}=1,2, \ldots, \mathrm{n})$, then the IGS-IVIFHCAA operator reduces to be the induced generalized Shapley Atanassov's intuitionistic fuzzy hybrid Choquet arithmetical averaging (IGS-IFHCAA) operator, denoted by

$$
\begin{aligned}
& \operatorname{IGS}-I F H C A A_{\Phi, \varphi}\left(<u_{1}, \tilde{\alpha}_{1}>,<u_{2}, \tilde{\alpha}_{2}>, \ldots,<u_{n}, \tilde{\alpha}_{n}>\right) \\
& =\frac{\bigoplus_{j=1}^{\mathrm{n}}\left(\Phi_{\mathrm{A}_{(\mathrm{j})}}(\mu, \mathrm{N})-\Phi_{\mathrm{A}_{(\mathrm{j}+1)}}(\mu, \mathrm{N})\right) \varphi_{\tilde{a}_{(j)}}(\grave{v}, \tilde{\vartheta}) \tilde{\alpha}_{(j)}}{\sum_{\mathrm{j}=1}^{\mathrm{n}}\left(\Phi_{\mathrm{A}_{(j)}}(\mu, \mathrm{N})-\Phi_{\mathrm{A}_{(j+1)}}(\mu, \mathrm{N})\right) \varphi_{\tilde{a}_{(j)}}(\dot{\mathcal{V}}, \tilde{\vartheta})} \\
& =\left(1-\prod_{j=1}^{n}\left(1-a_{(j)}\right)^{\frac{\left(\Phi_{A_{(j)}}(\mu, \mathrm{N})-\Phi_{A_{(j+1)}}(\mu, \mathrm{N})\right) \varphi_{\left.\bar{a}_{(j)}\right)}(v, \tilde{\vartheta})}{\sum_{j=1}^{n}\left(\Phi_{A_{(j)}}(\mu, \mathrm{N})-\Phi_{A_{(j+1)}}(\mu, \mathrm{N})\right) \varphi_{\tilde{a}_{(j)}}(v, \tilde{\vartheta})}},\right. \\
& \left.\prod_{j=1}^{n} C_{(\mathrm{j})} \frac{\left(\Phi_{A_{(j)}}(\mu, \mathrm{N})-\Phi_{A_{(j+1)}}(\mu, \mathrm{N})\right) \varphi_{\bar{a}_{(j)}}(v, \tilde{\vartheta})}{\sum_{j=1}^{n}\left(\Phi_{A_{(j)}}(\mu, \mathrm{N})-\Phi_{A_{(j+1)}}(\mu, N)\right) \varphi_{\tilde{a}_{(j)}}(v, \tilde{\vartheta})}\right) .
\end{aligned}
$$

Remark 3.4 If $\varphi_{\tilde{a}_{i}}(\dot{v}, \tilde{\vartheta})=1 / n(i=1,2, \ldots, n)$, then the IGSIVIFHCAA operator degenerates to be the induced generalized Shapley Atanassov's interval-valued intuitionistic fuzzy Choquet arithmetical averaging (IGSIVIFCAA) operator 


$$
\begin{aligned}
& \operatorname{IGS-IVIFCAA~}_{\Phi}\left(<\mathrm{u}_{1}, \tilde{\alpha}_{1}>,<u_{2}, \tilde{\alpha}_{2}>, \ldots,<u_{n}, \tilde{\alpha}_{n}>\right) \\
& =\bigoplus_{\mathrm{j}=1}^{n}\left(\Phi_{\mathrm{A}_{(\mathrm{j})}}(\mu, \mathrm{N})-\Phi_{\mathrm{A}_{(\mathrm{j}+1)}}(\mu, \mathrm{N})\right) \tilde{\alpha}_{(j)} .
\end{aligned}
$$

Remark 3.5 If $\Phi_{\mathrm{A}_{(\mathrm{i})}}(\mu, \mathrm{N})-\Phi_{\mathrm{A}_{(\mathrm{i}+1)}}(\mu, \mathrm{N})=1 / \mathrm{n}(\mathrm{i}=1,2, \ldots, \mathrm{n})$, then the IGS-IVIFHCAA operator degenerates to be the induced Atanassov's interval-valued intuitionistic fuzzy Shapley averaging (I-IVIFSA) operator

$$
\begin{aligned}
& \text { I-IVIFSA }_{\varphi}\left(<\mathrm{u}_{1}, \tilde{\alpha}_{1}>,<u_{2}, \tilde{\alpha}_{2}>, \ldots,<u_{n}, \tilde{\alpha}_{n}>\right) \\
& =\bigoplus_{\mathrm{j}=1}^{n} \varphi_{\bar{a}_{(j)}}(v, \tilde{\vartheta}) \tilde{\alpha}_{(j)} .
\end{aligned}
$$

Furthermore, if $\mathrm{u}_{\mathrm{i}}=\tilde{\alpha}_{i}$, then we get the Atanassov's interval-valued intuitionistic fuzzy Shapley averaging (IVIFSA) operator

$$
\operatorname{IVIFSA}_{\varphi}\left(\tilde{\alpha}_{1}, \tilde{\alpha}_{2}, \ldots, \tilde{\alpha}_{n}\right)=\bigoplus_{i=1}^{\tilde{n}} \varphi_{\tilde{\alpha}_{i}}(v, \tilde{\vartheta}) \tilde{\alpha}_{i}
$$

which can be seen as an extension of the Shapley Atanassov's value-based intuitionistic fuzzy aggregation (SIFA) operator given by Zhang et al. [40].

Definition 3.2 An IGS-IVIFHCGM operator of dimension $n$ is a mapping IGS-IVIFHCGM: $\Omega^{n} \rightarrow \Omega$ defined on the set of second arguments of two tuples $<\mathrm{u}_{1}, \tilde{\alpha}_{1}>,<u_{2}, \tilde{\alpha}_{2}>, \ldots$, $<u_{n}, \tilde{\alpha}_{n}>$ with a set of order-inducing variables $u_{i}(i=1,2, \ldots$, $n)$, denoted by

$$
\begin{aligned}
& \operatorname{IGS-IVIFHCGM}_{\Phi, \varphi}\left(<\mathrm{u}_{1}, \tilde{\alpha}_{1}>,<u_{2}, \tilde{\alpha}_{2}>, \ldots,<u_{n}, \tilde{\alpha}_{n}>\right) \\
& =\bigotimes_{j=1}^{n} \tilde{\alpha}_{(j)} \frac{\left(\Phi_{A_{(j)}}(\mu, \mathrm{N})-\Phi_{A_{(j+1)}}(\mu, \mathrm{N})\right) \varphi_{\bar{a}_{(j)}}(v, \tilde{\vartheta})}{\sum_{j=1}^{n}\left(\Phi_{A_{(j)}}(\mu, \mathrm{N})-\Phi_{A_{(j+1)}}(\mu, \mathrm{N})\right) \varphi_{\bar{q}_{(j)}}(v, \overline{\tilde{g}})}
\end{aligned}
$$

where the notations as given in Definition 3.1.

Theorem 3.3 Let $\tilde{\alpha}_{i}=\left(\left[a_{i}, b_{i}\right],\left[c_{\mathrm{i}}, \mathrm{d}_{\mathrm{i}}\right]\right)(\mathrm{i}=1,2, \ldots, \mathrm{n})$ be a collection of IVIFVs in $\Omega$, then their aggregated value by using the IGS-IVIFHCGM operator is also an IVIFV, denoted by

$$
\operatorname{IGS}-I V I F H C G M_{\Phi, \varphi}\left(<u_{1}, \tilde{\alpha}_{1}>,<u_{2}, \tilde{\alpha}_{2}>, \ldots,<u_{n}, \tilde{\alpha}_{n}>\right)
$$$$
=\left(\left[\prod_{j=1}^{n} a_{(j)} \frac{\left(\Phi_{A_{(j)}}(\mu, N)-\Phi_{A_{(j+1)}}(\mu, \mathrm{N})\right) \varphi_{a_{(j)}}(v, \tilde{g})}{\sum_{j=1}^{n}\left(\Phi_{A_{(j)}}(\mu, \mathrm{N})-\Phi_{A_{(j+1)}}(\mu, \mathrm{N})\right) \varphi_{\tilde{a}_{(j)}}(v, \tilde{\vartheta})},\right.\right.
$$

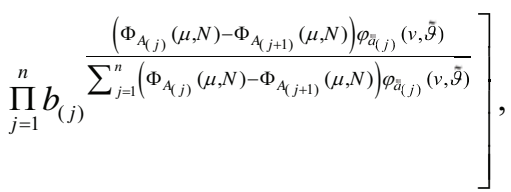

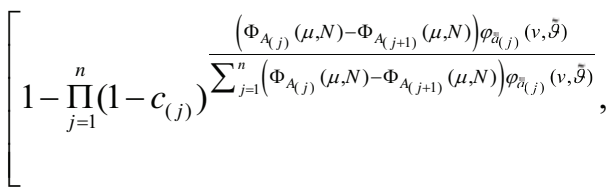

$$
\begin{aligned}
& \left.\left.1-\prod_{j=1}^{n}\left(1-d_{(j)}\right)^{\frac{\left(\Phi_{A_{(j)}}(\mu, \mathrm{N})-\Phi_{A_{(j+1)}}(\mu, \mathrm{N})\right) \varphi_{a_{(j)}}(v, \dot{\vartheta})}{\sum_{j=1}^{n}\left(\Phi_{A_{(j)}}(\mu, \mathrm{N})-\Phi_{A_{(j+1)}}(\mu, \mathrm{N})\right) \varphi_{\bar{a}_{(j)}}(v, \tilde{\vartheta})}}\right]\right) .
\end{aligned}
$$

Proof. The proof of Theorem 3.3 is similar to that of Theorem 3.2.

Remark 3.6 If $\mu$ and $v$ are both additive, then the IGSIVIFHCGM operator reduces to be the induced Atanassov's interval-valued intuitionistic fuzzy hybrid weighted geometric mean (I-IVIFHWGM) operator

$$
\begin{aligned}
& \text { I-IVIFHWGM }{ }_{\mu, \mathrm{v}}\left(<\mathrm{u}_{1}, \tilde{\alpha}_{1}>,<u_{2}, \tilde{\alpha}_{2}>, \ldots,<u_{n}, \tilde{\alpha}_{n}>\right) \\
& =\bigotimes_{\mathrm{j}=1}^{n} \tilde{\alpha}_{(j)} \frac{\mathrm{w}_{\mathrm{j}} \omega_{(\mathrm{j})}}{\sum_{j=1}^{n} w_{j} \omega_{(j)}},
\end{aligned}
$$

where $\mu(\mathrm{i})=\mathrm{w}_{\mathrm{i}}$ and $\mathrm{v}\left(\tilde{\alpha}_{i}\right)=\omega_{i}(\mathrm{i}=1,2, \ldots, \mathrm{n})$.

R emark 3.7 If $\mathrm{u}_{\mathrm{i}}=\tilde{\alpha}_{i}^{\varphi_{\bar{a}_{i}}(v, \tilde{\vartheta})}(\mathrm{i}=1,2, \ldots, \mathrm{n})$, the IGS-IVIFHCGM operator reduces to be the generalized Shapley Atanassov's interval-valued intuitionistic fuzzy hybrid Choquet geometric mean (GS-IVIFHCGM) operator

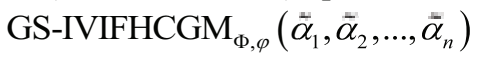

$$
\begin{aligned}
& =\bigotimes_{\mathrm{j}=1}^{n} \tilde{\alpha}_{(j)} \frac{\left(\Phi_{A_{(\mathrm{j})}}(\mu, \mathrm{N})-\Phi_{A_{(j+1)}}(\mu, \mathrm{N})\right) \varphi_{\tilde{a}_{(j)}}(v, \tilde{g})}{\sum_{j=1}^{n}\left(\Phi_{A_{(j)}}(\mu, \mathrm{N})-\Phi_{A_{(j+1)}}(\mu, \mathrm{N})\right) \varphi_{a_{(j)}}(v, \tilde{g})},
\end{aligned}
$$

where $\tilde{\alpha}_{(j)}{ }^{\varphi_{\hat{a}_{(j)}}(v, \bar{\vartheta})}$ is the jth largest of $\tilde{\alpha}_{i}^{\varphi_{\bar{a}_{i}}^{(v, \tilde{\vartheta})}}(\mathrm{i}=1,2, \ldots, \mathrm{n})$.

R emark 3.8 If each IVIFV $\tilde{\alpha}_{i}=\left(\left[a_{i}, b\right],\left[\mathrm{c}_{\mathrm{i}}, \mathrm{d}_{\mathrm{i}}\right]\right)(\mathrm{i}=1,2, \ldots, \mathrm{n})$ degenerates to be an Atanassov's intuitionistic fuzzy value, namely, $\tilde{\alpha}_{i}=\left(a_{i}, c_{i}\right)(\mathrm{i}=1,2, \ldots, \mathrm{n})$, then the IGS-IVIFHCGM 
F anyong M eng et al.

operator reduces to be the induced generalized Shapley Atanassov's intuitionistic fuzzy hybrid Choquet geometric mean (IGS-IFHCGM) operator, denoted by

$$
\begin{aligned}
& \operatorname{IGS}-I F H C G M_{\Phi, \varphi}\left(<u_{1}, \tilde{\alpha}_{1}>,<u_{2}, \tilde{\alpha}_{2}>, \ldots,<u_{n}, \tilde{\alpha}_{n}>\right) \\
& =\bigotimes_{\mathrm{j}=1}^{\mathrm{n}} \tilde{\alpha}_{(j)} \frac{\left(\Phi_{\mathrm{A}_{(\mathrm{j})}}(\mu, \mathrm{N})-\Phi_{\mathrm{A}_{(\mathrm{j}+1)}}(\mu, \mathrm{N})\right) \varphi_{\bar{a}_{(j)}}(v, \tilde{\vartheta})}{\sum_{j=1}^{n}\left(\Phi_{A_{(j)}}(\mu, \mathrm{N})-\Phi_{A_{(\mathrm{j}+1)}}(\mu, \mathrm{N})\right) \varphi_{a_{(j)}}(v, \tilde{\vartheta})}
\end{aligned}
$$

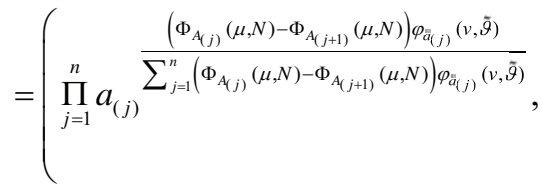

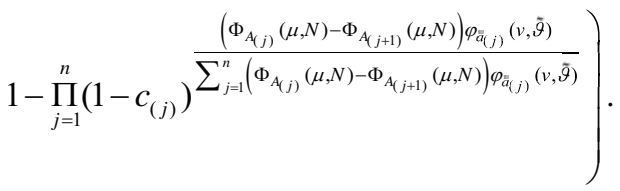

Remark 3.9 If $\varphi_{\tilde{a}_{i}}(v, \tilde{\vartheta})=1 / n(i=1,2, \ldots, \mathrm{n})$, then the IGSIVIFHCGM operator degenerates to be the induced generalized Shapley Atanassov's interval-valued intuitionistic fuzzy Choquet geometric mean (IGSIVIFCGM) operator

$$
\begin{aligned}
& \operatorname{IGS}_{\operatorname{IVIFCGM}}\left(<\mathrm{u}_{1}, \tilde{\alpha}_{1}>,<u_{2}, \tilde{\alpha}_{2}>, \ldots,<u_{n}, \tilde{\alpha}_{n}>\right) \\
& =\bigotimes_{\mathrm{j}=1}^{\mathrm{n}} \tilde{\alpha}_{(j)}\left(\Phi_{A_{(j)}}(\mu, N)-\Phi_{A_{(j+1)}}(\mu, \mathrm{N})\right) \text {. }
\end{aligned}
$$

Remark 3.10 If $\Phi_{A_{(i)}}(\mu, \mathrm{N})-\Phi_{A_{(i+1)}}(\mu, \mathrm{N})=1 / n(i=1,2, \ldots, n)$, then the IGS-IVIFHCGM operator degenerates to be the induced Shapley Atanassov's interval-valued intuitionistic fuzzy geometric mean (IS-IVIFGM) operator

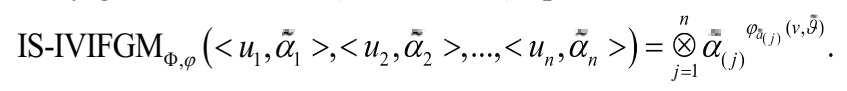

Furthermore, if $u_{\mathrm{i}}=\tilde{\alpha}_{i}$, then we get the Shapley Atanassov's interval-valued intuitionistic fuzzy geometric (S-IVIFG) operator

$$
\mathrm{S}-\mathrm{IVIFG} \mathrm{I}_{\Phi, \varphi}\left(\tilde{\alpha}_{1}, \tilde{\alpha}_{2}, \ldots, \tilde{\alpha}_{n}\right)=\bigotimes_{i=1}^{\bar{n}} \tilde{\alpha}_{i}^{\varphi_{\bar{q}_{i}}(v, \bar{\vartheta})} .
$$

\subsection{Some desirable properties}

Proposition 3.3 (Commutativity) Let $\tilde{\alpha}_{i}=\left(\left[a_{i}, b_{i}\right],\left[\mathrm{c}_{\mathrm{i}}, \mathrm{d}_{\mathrm{i}}\right]\right)$ $(i=1,2, \ldots, n)$ be a collection of IVIFVs in $\Omega$, and $\tilde{\alpha}_{i}^{\prime}=$ $\left(\left[a_{i}^{\prime}, b_{i}^{\prime}\right],\left[c_{i}^{\prime}, d_{i}^{\prime}\right]\right)(i=1,2, \ldots, n)$ be a permutation of $\tilde{\alpha}_{i}$. Then,

$$
\begin{aligned}
& \operatorname{IGS-IVIFHCAA}_{\Phi, \varphi}\left(<\mathrm{u}_{1}, \tilde{\alpha}_{1}>, \ldots,<u_{n}, \tilde{\alpha}_{n}>\right) \\
& =\operatorname{IGS}-I V I F H C A A_{\Phi, \varphi}\left(<\mathrm{u}_{1}, \tilde{\alpha}_{1}^{\prime}>, \ldots,<u_{n}, \tilde{\alpha}_{n}^{\prime}>\right) \text {, } \\
& \operatorname{IGS-IVIFHCGM}_{\Phi, \varphi}\left(<\mathrm{u}_{1}, \tilde{\alpha}_{1}>, \ldots,<u_{n}, \tilde{\alpha}_{n}>\right)
\end{aligned}
$$

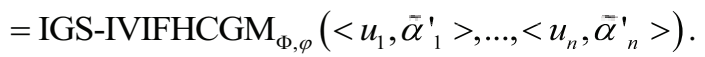

Proof. From Definitions 3.1 and 3.2, it is not difficult to get the conclusion.

Proposition 3.4 (M onotonicity) Let $\tilde{\alpha}_{i}=\left(\left[a_{i}, b_{\mathrm{i}}\right],\left[\mathrm{c}_{\mathrm{i}}, \mathrm{d}_{\mathrm{i}}\right]\right)$ and $\bar{\beta}_{i}=\left(\left[e_{i}, f_{i}\right],\left[g_{i}, h_{i}\right]\right)(i=1,2, \ldots, n)$ be two collections of IVIFVs in $\Omega$. If $\tilde{\alpha}_{i} \leq \tilde{\beta}_{i}$ for each $i=1,2, \ldots, n$, and $\mathrm{v}(\tilde{S})=\mathrm{v}(\tilde{\Gamma})$ for all $\tilde{S} \subseteq \tilde{\vartheta}$ and $\tilde{T} \subseteq \tilde{\theta}$ with $\left\{\mathrm{j} \mid \tilde{\alpha}_{j} \in \tilde{S}, j=1,2, \ldots, n\right\}=\left\{j \mid \tilde{\beta}_{i} \in\right.$ $\bar{\Gamma}, \mathrm{j}=1,2, \ldots, \mathrm{n}\}$, where $\mathrm{v}$ is a fuzzy measure on $\bar{\vartheta}=\left\{\bar{\alpha}_{j}\right\}_{j=1, \ldots, n}$ and $\bar{\theta}=\left\{\bar{\beta}_{j}\right\}_{i=1, \ldots, n}$. Then,

$$
\begin{aligned}
& \operatorname{IGS-IVIFHCAA}_{\Phi, \varphi}\left(<\mathrm{u}_{1}, \tilde{\alpha}_{1}>, \ldots,<u_{n}, \tilde{\alpha}_{n}>\right) \\
& \leq \operatorname{IGS-IVIFHCAA}_{\Phi, \varphi}\left(<\mathrm{u}_{1}, \tilde{\beta}_{1}>, \ldots,<u_{n}, \tilde{\beta}_{n}>\right), \\
& \operatorname{IGS-IVIFHCGM}_{\Phi, \varphi}\left(<\mathrm{u}_{1}, \tilde{\alpha}_{1}>, \ldots,<u_{n}, \tilde{\alpha}_{n}>\right) \\
& \leq \operatorname{IGS}-I V I F H C G M_{\Phi, \varphi}\left(<u_{1}, \tilde{\beta}_{1}>, \ldots,<u_{n}, \tilde{\beta}_{n}>\right) .
\end{aligned}
$$

Proof. For Eq.(7): Let

$$
\begin{array}{r}
\text { IGS-IVIFHCAA }_{\Phi, \varphi}\left(<\mathrm{u}_{1}, \tilde{\alpha}_{1}>, \ldots,<u_{n}, \tilde{\alpha}_{n}>\right) \\
=\frac{\bigoplus_{\mathrm{j}=1}^{\mathrm{n}}\left(\Phi_{\mathrm{A}_{(j)}}(\mu, \mathrm{N})-\Phi_{\mathrm{A}_{(j+1)}}(\mu, \mathrm{N})\right) \varphi_{\tilde{a}_{(j)}}(\nu, \tilde{\vartheta}) \tilde{\alpha}_{(j)}}{\sum_{\mathrm{j}=1}^{\mathrm{n}}\left(\Phi_{\mathrm{A}_{(\mathrm{j})}}(\mu, \mathrm{N})-\Phi_{\mathrm{A}_{(j+1)}}(\mu, \mathrm{N})\right) \varphi_{\tilde{a}_{(j)}}(\mathcal{v}, \tilde{\vartheta})}
\end{array}
$$

and

$$
\begin{gathered}
\operatorname{IGS} \operatorname{IVIFHCAA}_{\Phi, \varphi}\left(<\mathrm{u}_{1}, \tilde{\beta}_{1}>, \ldots,<u_{n}, \tilde{\beta}_{n}>\right) \\
=\frac{\bigoplus_{j=1}^{\mathrm{n}}\left(\Phi_{\mathrm{A}_{(\mathrm{j})}}(\mu, \mathrm{N})-\Phi_{\mathrm{A}_{(j+1)}}(\mu, \mathrm{N})\right) \varphi_{\tilde{\beta}_{(j)}}(\dot{v}, \tilde{\theta}) \tilde{\beta}_{(i)}}{\sum_{\mathrm{j}=1}^{\mathrm{n}}\left(\Phi_{\mathrm{A}_{(\mathrm{j})}}(\mu, \mathrm{N})-\Phi_{\mathrm{A}_{(j+1)}}(\mu, \mathrm{N})\right) \varphi_{\tilde{\beta}_{(i)}}(\dot{v}, \tilde{\theta})} .
\end{gathered}
$$

From $\mathrm{v}(\tilde{S})=\mathrm{v}(\tilde{T})$ for all $\tilde{S} \subseteq \tilde{\vartheta}$ and $\tilde{\Gamma} \subseteq \tilde{\theta}$ with $\left\{j \mid \tilde{\alpha}_{j} \in \tilde{S}\right.$, $\mathrm{j}=1,2, \ldots, \mathrm{n}\}=\left\{\mathrm{j} \mid \tilde{\beta}_{i} \in \tilde{\Gamma}, \mathrm{j}=1,2, \ldots, \mathrm{n}\right\}$, we get $\varphi_{\tilde{\alpha}_{i}}(\dot{v}, \tilde{\vartheta})=$ 
$\varphi_{\tilde{\beta}_{i}}(\dot{v}, \tilde{\theta}) \geq 0$ for all $\mathrm{i}=1,2, \ldots, \mathrm{n}$. Since $\tilde{\alpha}_{i} \leq \tilde{\vartheta}_{i}$ for all $\mathrm{i}=1,2, \ldots, \mathrm{n}$, we obtain

$$
\begin{aligned}
& \frac{\left(\Phi_{\mathrm{A}_{(\mathrm{j})}}(\mu, \mathrm{N})-\Phi_{\mathrm{A}_{(j+1)}}(\mu, \mathrm{N})\right) \varphi_{\tilde{a}_{(j)}}(\nu, \tilde{\vartheta}) \tilde{\alpha}_{(j)}}{\sum_{\mathrm{j}=1}^{\mathrm{n}}\left(\Phi_{\mathrm{A}_{(\mathrm{j})}}(\mu, \mathrm{N})-\Phi_{\mathrm{A}_{(\mathrm{j}+1)}}(\mu, \mathrm{N})\right) \varphi_{\tilde{a}_{(j)}}(\dot{v}, \tilde{\vartheta})} \\
& \leq \frac{\left(\Phi_{\mathrm{A}_{(j)}}(\mu, \mathrm{N})-\Phi_{\mathrm{A}_{(\mathrm{j}+1)}}(\mu, \mathrm{N})\right) \varphi_{\tilde{\beta}_{(i)}}(\dot{v}, \tilde{\theta}) \tilde{\beta}_{(i)}}{\sum_{\mathrm{j}=1}^{\mathrm{n}}\left(\Phi_{\mathrm{A}_{(j)}}(\mu, \mathrm{N})-\Phi_{\mathrm{A}_{(\mathrm{j}+1)}}(\mu, \mathrm{N})\right) \varphi_{\tilde{\beta}_{(i)}}(\dot{v}, \tilde{\theta})}
\end{aligned}
$$

for all $j=1,2, \ldots, n$.

Thus,

$$
\begin{aligned}
& \operatorname{IGS} \operatorname{IVIFHCAA}_{\Phi, \varphi}\left(<\mathrm{u}_{1}, \tilde{\alpha}_{1}>, \ldots,<u_{n}, \tilde{\alpha}_{n}>\right)
\end{aligned}
$$

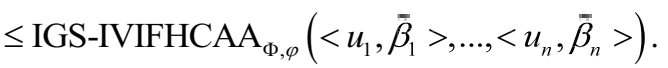

Similarly, one can get Eq.(8).

Proposition 3.5 (Idempotency) Let $\tilde{\alpha}_{i}=\left(\left[a_{i}, b_{i}\right],\left[c_{i}, \mathrm{~d}_{\mathrm{i}}\right]\right)$ $(i=1,2, \ldots, n)$ be a collection of IVIFVs in $\Omega$. If $\tilde{\alpha}_{i}=\tilde{\alpha}=$ $([e, f],[g, h])$ for all $i=1,2, \ldots, n$, then

$$
\begin{aligned}
& \operatorname{IGS} \text { IVIFHCAA } \\
& \operatorname{IGS}, \varphi\left(<\mathrm{u}_{1}, \tilde{\alpha}_{1}>, \ldots,<u_{n}, \tilde{\alpha}_{n}>\right)=\tilde{\alpha}, \\
&
\end{aligned}
$$

Proof. For Eq.(9): From Theorem 3.2, we have IGS-IVIFHCAA $_{\Phi, \varphi}\left(<\mathrm{u}_{1}, \tilde{\alpha}_{1}>, \ldots,<u_{n}, \tilde{\alpha}_{n}>\right)$

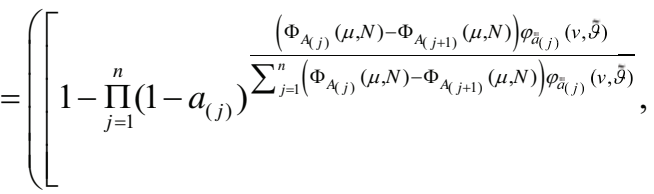

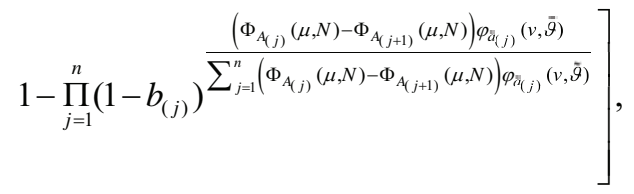

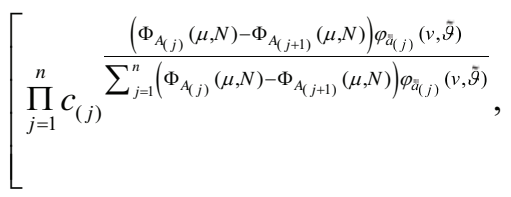

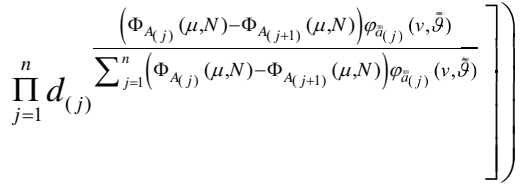

$$
\begin{aligned}
& =\left(\left[1-\prod_{j=1}^{n}(1-a)^{\frac{\left(\Phi_{A_{(j)}}(\mu, \mathrm{N})-\Phi_{A_{(j+1)}}(\mu, \mathrm{N})\right) \varphi_{\bar{a}_{(j)}}(v, \tilde{\vartheta})}{\sum_{j=1}^{n}\left(\Phi_{A_{(j)}}(\mu, \mathrm{N})-\Phi_{A_{(j+1)}}(\mu, \mathrm{N})\right) \varphi_{\tilde{a}_{(j)}}(v, \tilde{\vartheta})}},\right.\right. \\
& \left.1-\prod_{j=1}^{n}(1-b)^{\frac{\left(\Phi_{A_{(j)}}(\mu, \mathrm{N})-\Phi_{A_{(j+1)}}(\mu, N)\right) \varphi_{\hat{a_{(j)}}}(v, \tilde{\vartheta})}{\sum_{j=1}^{n}\left(\Phi_{A_{(j)}}(\mu, \mathrm{N})-\Phi_{A_{(j+1)}}(\mu, \mathrm{N})\right) \varphi_{\hat{a}(j)}(v, \tilde{q})}}\right],
\end{aligned}
$$

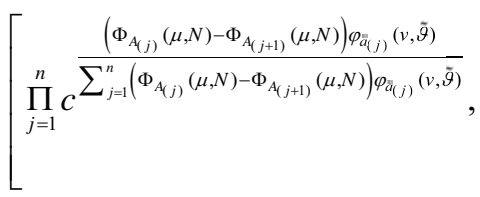

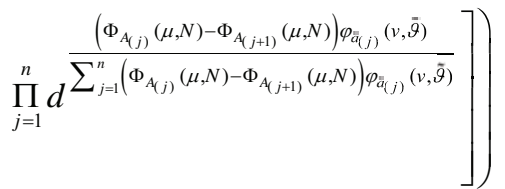

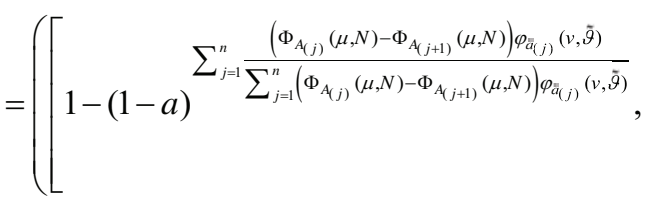

$$
\begin{aligned}
& \left.1-(1-b) \sum_{j=1}^{n} \frac{\left(\Phi_{A_{(j)}}(\mu, \mathrm{N})-\Phi_{A_{(j+1)}}(\mu, N)\right) \varphi_{\tilde{a}_{(j)}}(v, \tilde{\vartheta})}{\sum_{j=1}^{n}\left(\Phi_{A_{(j)}}(\mu, N)-\Phi_{A_{(j+1)}}(\mu, \mathrm{N})\right) \varphi_{\bar{a}_{(j)}}(v, \tilde{\vartheta})}\right], \\
& {\left[\sum^{\sum_{j=1}^{n} \frac{\left(\Phi_{A_{(j)}}(\mu, \mathrm{N})-\Phi_{A_{(j+1)}}(\mu, \mathrm{N})\right) \varphi_{\left.a_{(j)}\right)}(v, \tilde{\vartheta})}{\sum_{j=1}^{n}\left(\Phi_{A_{(j)}}(\mu, \mathrm{N})-\Phi_{A_{(j+1)}}(\mu, \mathrm{N})\right) \varphi_{\tilde{a}_{(j)}}(v, \tilde{\vartheta})}},\right.}
\end{aligned}
$$

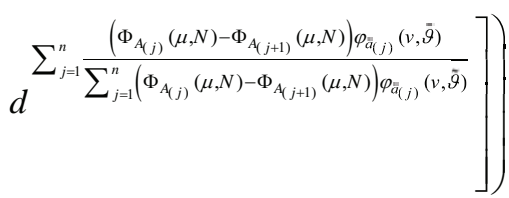

$$
\begin{aligned}
& =([\mathrm{a}, \mathrm{b}],[\mathrm{c}, \mathrm{d}])
\end{aligned}
$$


F anyong M eng et al.

$=\tilde{\alpha}$.

From Theorem 3.3 and the proof of Eq.(9), it is not difficult to get Eq.(10).

Proposition 3.6 (B oundary) Let $\tilde{\alpha}_{i}=\left(\left[a_{i}, b_{i}\right],\left[c_{i}, \mathrm{~d}_{\mathrm{i}}\right]\right)(\mathrm{i}=1,2$, $\ldots, n)$ be a collection of IVIFVs in $\Omega$, then

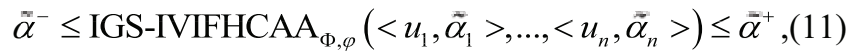

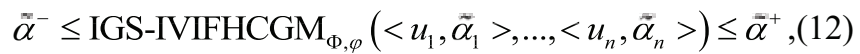
where

$$
\tilde{\alpha}^{-}=\left(\left[\min _{j} a_{j}, \min _{j} b_{j}\right],\left[\max _{j} c_{j}, \max _{j} d_{j}\right]\right)
$$

and

$$
\tilde{\alpha}^{+}=\left(\left[\max _{j} a_{j}, \max _{j} b_{j}\right],\left[\min _{j} c_{j}, \min _{j} d_{j}\right]\right) .
$$

Proof. From Proposition 3.4, we have

$$
\begin{aligned}
& \operatorname{IGS-IVIFHCAA~}_{\Phi, \varphi}\left(<\mathrm{u}_{1}, \bar{\alpha}^{-}>, \ldots,<u_{n}, \tilde{\alpha}^{-}>\right)
\end{aligned}
$$

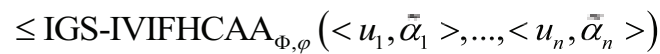

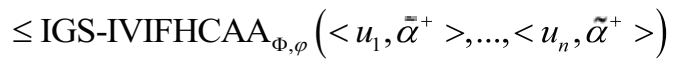

and

$$
\begin{aligned}
& \operatorname{IGS}-I V I F H C G M_{\Phi, \varphi}\left(<u_{1}, \tilde{\alpha}^{-}>, \ldots,<u_{n}, \tilde{\alpha}^{-}>\right) \\
& \leq \operatorname{IGS}-I V I F H C G M_{\Phi, \varphi}\left(<\mathrm{u}_{1}, \tilde{\alpha}_{1}>, \ldots,<u_{n}, \tilde{\alpha}_{n}>\right)
\end{aligned}
$$

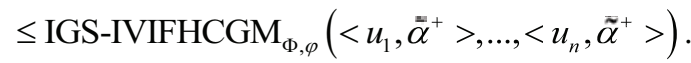

By Proposition 3.5, we get

$$
\begin{aligned}
& \operatorname{IGS} \operatorname{IVIFHCAA}_{\Phi, \varphi}\left(<\mathrm{u}_{1}, \tilde{\alpha}^{-}>, \ldots,<u_{n}, \tilde{\alpha}^{-}>\right) \\
= & \operatorname{IGS}^{-I V I F H C G M} \\
= & \tilde{\alpha}^{-}
\end{aligned}
$$

and

$$
\begin{aligned}
& \operatorname{IGS} \operatorname{IVIFHCAA}_{\Phi, \varphi}\left(<\mathrm{u}_{1}, \tilde{\alpha}^{+}>, \ldots,<u_{n}, \tilde{\alpha}^{+}>\right) \\
& =\operatorname{IGS-IVIFHCGM}_{\Phi, \varphi}\left(<\mathrm{u}_{1}, \bar{\alpha}^{+}>, \ldots,<u_{n}, \overline{\tilde{\alpha}}^{+}>\right) \\
& =\tilde{\alpha}^{+} .
\end{aligned}
$$

From above, the conclusion is obtained.

Example 3.2 In Example 3.1, if the IGS-IVIFHCAA operator is applied to calculate the comprehensive intervalvalued intuitionistic fuzzy value, then it has

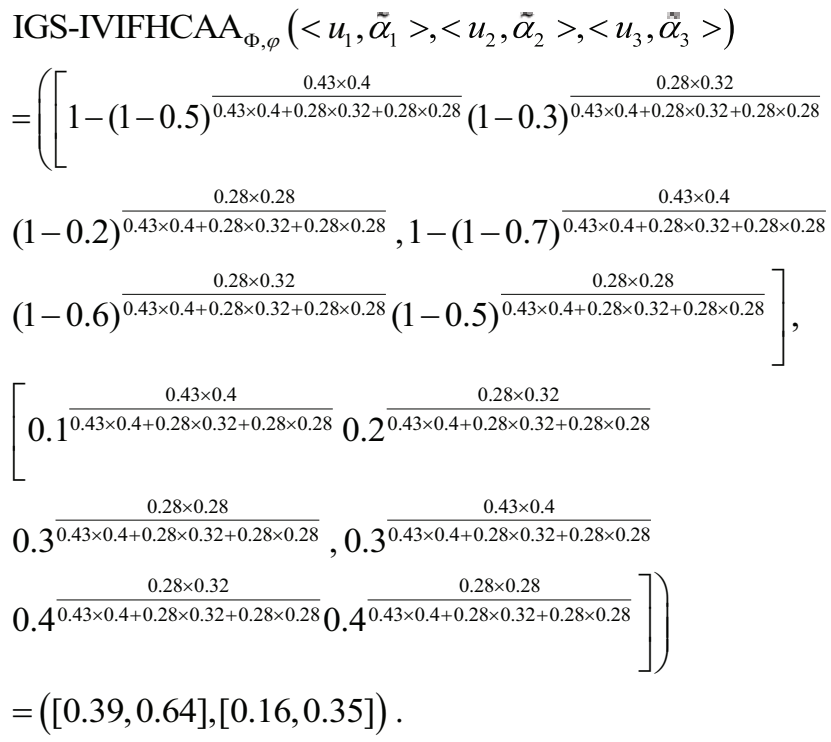

If the IGS-IVIFHCGM operator is applied to calculate the comprehensive interval-valued intuitionistic fuzzy value, then it has

$$
\begin{aligned}
& \operatorname{IGS-IVIFHCGM}_{\Phi, \varphi}\left(<\mathrm{u}_{1}, \tilde{\alpha}_{1}>,<u_{2}, \tilde{\alpha}_{2}>,<u_{3}, \tilde{\alpha}_{3}>\right) \\
& =\left[0.5^{\frac{0.43 \times 0.4}{0.43 \times 0.4+0.28 \times 0.32+0.28 \times 0.28}} 0.3^{\frac{0.28 \times 0.32}{0.43 \times 0.4+0.28 \times 0.32+0.28 \times 0.28}}\right. \\
& 0.2^{\frac{0.28 \times 0.28}{0.43 \times 0.4+0.28 \times 0.32+0.28 \times 0.28}}, 0.7^{\frac{0.43 \times 0.4}{0.43 \times 0.4+0.28 \times 0.32+0.28 \times 0.28}} \\
& \left.0.6^{\frac{0.28 \times 0.32}{0.43 \times 0.4+0.28 \times 0.32+0.28 \times 0.28}} 0.5^{\frac{0.28 \times 0.28}{0.43 \times 0.4+0.28 \times 0.32+0.28 \times 0.28}}\right] \text {, } \\
& {\left[1-(1-0.1)^{\frac{0.43 \times 0.4}{0.43 \times 0.4+0.28 \times 0.32+0.28 \times 0.28}} 1-(1-0.2)^{\frac{0.28 \times 0.32}{0.43 \times 0.4+0.28 \times 0.32+0.28 \times 0.28}}\right.} \\
& (1-0.3)^{\frac{0.28 \times 0.28}{0.43 \times 0.4+0.28 \times 0.32+0.28 \times 0.28}}, 1-(1-0.3)^{\frac{0.43 \times 0.4}{0.43 \times 0.4+0.28 \times 0.32+0.28 \times 0.28}} \\
& \left.\left.(1-0.4)^{\frac{0.28 \times 0.32}{0.43 \times 0.4+0.28 \times 0.32+0.28 \times 0.28}}(1-0.4)^{\frac{0.28 \times 0.28}{0.43 \times 0.4+0.28 \times 0.32+0.28 \times 0.28}}\right]\right) \\
& =([0.35,0.62],[0.18,0.35]) \text {. }
\end{aligned}
$$




\section{An approach to Atanassov's interval-valued intuitionistic fuzzy multi-attribute decision making}

\subsection{A special case of the IGS-IVIFHCAA and IGS- IVIFHCGM operators}

From Definition 2.6, we know the fuzzy measure is defined on the power set, which makes the problem exponentially complex. Thus, it is not easy to get the fuzzy measure of each combination in a set when it is large. The $\lambda$-fuzzy measure proposed by Seguno [47] seems to well deal with this issue, which is expressed by

$$
g_{\lambda}(A \cup B)=g_{\lambda}(A)+g_{\lambda}(B)+\lambda g_{\lambda}(A) g_{\lambda}(B),
$$

where $\lambda>-1$, and $A, B \subseteq N$ with $A \cap B=\varnothing$.

It is apparent that when $\lambda=0$, then $g_{\lambda}$ is an additive measure, which means there is no interaction between coalitions $A$ and $B$. If $\lambda>0$, then $g_{\lambda}$ is called a superadditive measure, which reflects there exists complementary interaction between coalitions $A$ and $B$. If $-1<\lambda<0$, then $g_{\lambda}$ is said to be a subadditive measure, which shows there exists redundancy interaction between coalitions $A$ and B.

For finite set $N$, the $\lambda$-fuzzy measure $g_{\lambda}$ can be equivalently expressed by

$$
g_{\lambda}(A)=\left\{\begin{array}{cl}
\frac{1}{\lambda}\left(\prod_{i \in A}\left[1+\lambda g_{\lambda}(i)\right]-1\right) & \text { if } \lambda \neq 0 \\
\sum_{i \in A} g_{\lambda}(i) & \text { if } \lambda=0
\end{array} .\right.
$$

From $\mu(\mathrm{N})=1$, we know $\lambda$ is determined by $\prod_{i \in \mathbb{N}}\left[1+\lambda g_{\lambda}(i)\right]=1+\lambda$. So when each $g_{\lambda}(i)$ is given, we can get the value of $\lambda$. From Eq.(14), for the set $N$ with $n$ elements we only need $n$ values to get the fuzzy measure of each subset in N. Furthermore, if $\sum_{i=1}^{n} g_{\lambda}(i)=1$, then $\lambda=0$.

Based on the $\lambda$-fuzzy measure, we further define the induced generalized $\lambda$-Shapley Atanassov's interval-valued intuitionistic fuzzy hybrid Choquet arithmetical averaging (IG $\lambda \mathrm{S}$ - IVIFHCAA) operator and the induced generalized $\lambda$ - Shapley Atanassov's interval-valued intuitionistic fuzzy hybrid Choquet geometric mean (IG $\lambda$ S-IVIFHCGM) operator as follows:

The IG $\lambda$ S-IVIFHCAA operator

$$
\begin{aligned}
& \text { IG } \lambda \text { S-IVIFHCAA }{ }_{\Phi, \varphi}\left(<\mathrm{u}_{1}, \tilde{\alpha}_{1}>, \ldots,<u_{n}, \tilde{\alpha}_{n}>\right) \\
& =\frac{\bigoplus_{j=1}^{n}\left(\Phi_{\mathrm{A}_{(\mathrm{j})}}\left(\mathrm{g}_{\lambda}, \mathrm{N}\right)-\Phi_{\mathrm{A}_{(j+1)}}\left(\mathrm{g}_{\lambda}, \mathrm{N}\right)\right) \varphi_{\tilde{a}_{(j)}}\left(\tilde{g}_{\lambda^{\prime}}, \tilde{\vartheta}\right) \tilde{\alpha}_{(j)}}{\sum_{\mathrm{j}=1}^{\mathrm{n}}\left(\Phi_{\mathrm{A}_{(\mathrm{j})}}\left(\mathrm{g}_{\lambda}, \mathrm{N}\right)-\Phi_{\mathrm{A}_{(j+1)}}\left(\mathrm{g}_{\lambda}, \mathrm{N}\right)\right) \varphi_{\tilde{a}_{(j)}}\left(\tilde{g}_{\lambda^{\prime}}, \tilde{\vartheta}\right)},
\end{aligned}
$$

and the IG $\lambda$ S-IVIFHCGM operator

$$
\begin{aligned}
& \operatorname{IG} \lambda \mathrm{S}-\mathrm{IVIFHCGM}_{\Phi, \varphi}\left(<\mathrm{u}_{1}, \tilde{\alpha}_{1}>, \ldots,<u_{n}, \tilde{\alpha}_{n}>\right)
\end{aligned}
$$

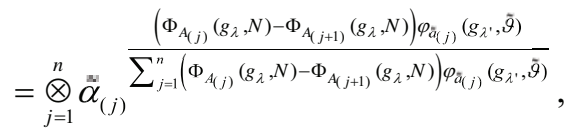

where $\Phi_{A(j)}\left(g_{\lambda}, N\right)$ is the generalized Shapley value w.r.t. the $\lambda$-fuzzy measure $g_{\lambda}$ on ordered set $N=\{1,2, \ldots, n\}$ for $A_{(j)}=$ $\{j, \ldots, n\}$ with $A_{(n+1)}=\varnothing$, and $\varphi_{\tilde{a}_{i}}\left(\tilde{g}_{\lambda^{\prime}}, \tilde{\vartheta}\right)$ is the Shapley value w.r.t. the $\lambda$-fuzzy measure $g_{\lambda^{\prime}}$ on $\bar{\vartheta}=\left\{\tilde{\alpha}_{i}\right\}_{i \in N}$ for $\tilde{\alpha}_{i}(\mathrm{i}=1,2, \ldots$, n).

\subsection{The models for the optimal $\lambda-f u z z y$ measures}

Now, consider a multi-attribute decision making problem under Atanassov's interval-valued intuitionistic fuzzy environment. Let $A=\left\{a_{1}, a_{2}, \ldots, a_{m}\right\}$ be the set of alternatives, and $C=\left\{C_{1}, C_{2}, \ldots, C_{n}\right\}$ be the set of attributes. Assume that $\left[a_{i j}, b_{i j}\right]$ and $\left[c_{i j}, d_{i j}\right]$ are the interval degrees of membership and non-membership of the alternative $a_{i}$ w.r.t. the criterion $c_{j}$, respectively, where $\left[a_{i j}, b_{i j}\right] \subseteq[0,1]$ and $\left[c_{i j}, d_{i j}\right] \subseteq[0,1]$ with $b_{i j}+d_{i j} \leq 1$. In other words, the evaluation of the alternative $a_{i}$ w.r.t. the attribute $c_{j}$ is an $\operatorname{IVIFV} \tilde{\alpha}_{i j}=\left(\left[a_{i j}, \mathrm{~b}_{\mathrm{ij}}\right],\left[\mathrm{c}_{\mathrm{ij}}, \mathrm{d}_{\mathrm{ij}}\right]\right) \quad(\mathrm{i}=1,2, \ldots, \mathrm{m} ; \mathrm{j}=1,2, \ldots, \mathrm{n})$. By $\tilde{A}=\left(\tilde{\alpha}_{i j}\right)_{m \times n}$, we denote the Atanassov's interval-valued intuitionistic fuzzy decision matrix. If all criteria $c_{j}$ $(j=1,2, \ldots, n)$ are benefits (i.e., the larger, the greater preference), then the criteria values do not need normalization. Otherwise, we normalize the IVIFV decision matrix $\tilde{A}=\left(\tilde{\alpha}_{i j}\right)_{m \times n}$ into $\tilde{R}=\left(\tilde{r}_{i j}\right)_{m \times n}$, where

$\tilde{r}_{i j}=\left\{\begin{array}{l}\tilde{\alpha}_{i j} \text { for benefit attribute } c_{j} \\ \left(\tilde{\alpha}_{i j}\right)^{C} \text { for cost attribute } c_{j}\end{array}\right.$ with $\tilde{r}_{i j}=\left(\left[e_{i j}, j_{i j}\right],\left[g_{i j}, h_{i j}\right]\right)$ $(i=1,2, \ldots, m ; j=1,2, \ldots, n)$ (see [15]).

Let $\tilde{\mathrm{R}}^{+}=\left(\bar{r}_{1}^{+}, \vec{r}_{2}^{+}, \ldots, \tilde{r}_{n}^{+}\right)$and $\tilde{R}^{-}=\left(\tilde{r}_{1}^{-}, \tilde{r}_{2}^{-}, \ldots, \bar{r}_{n}^{-}\right)$respectively 
F anyong M eng et al.

denote the positive and negative ideal vectors, where

$$
\begin{aligned}
& \tilde{r}_{j}^{+}=\left(\left[\max _{i} \mathrm{e}_{\mathrm{ij}}, \max _{\mathrm{i}} \mathrm{f}_{\mathrm{ij}}\right],\left[\min _{\mathrm{i}} \mathrm{g}_{\mathrm{ij}}, \min _{\mathrm{i}} \mathrm{h}_{\mathrm{ij}}\right]\right), \\
& \tilde{r}_{j}^{-}=\left(\left[\min _{i} \mathrm{e}_{\mathrm{ij}}, \min _{\mathrm{i}} \mathrm{f}_{\mathrm{ij}}\right],\left[\max _{\mathrm{i}} \mathrm{g}_{\mathrm{ij}}, \max _{\mathrm{i}} \mathrm{h}_{\mathrm{ij}}\right]\right)
\end{aligned}
$$

for all $\mathrm{j}=1,2, \ldots, n$.

Let

$$
d_{i j}=\frac{d_{i j}^{+}}{d_{i j}^{+}+d_{i j}^{-}},
$$

where

$$
\begin{aligned}
& d_{i j}^{+}=\frac{\left|e_{i j}-\max _{1 \leq i \leq m} e_{i j}\right|+\left|f_{i j}-\max _{1 \leq i \leq m} f_{i j}\right|+\left|g_{i j}-\min _{1 \leq i \leq m} g_{i j}\right|+\left|h_{i j}-\min _{1 \leq i \leq m} h_{i j}\right|}{4}, \\
& d_{i j}^{-}=\frac{\left|e_{i j}-\min _{1 \leq i \leq m} e_{i j}\right|+\left|f_{i j}-\min _{1 \leq i \leq m} f_{i j}\right|+\left|g_{i j}-\max _{1 \leq i \leq m} g_{i j}\right|+\left|h_{i j}-\max _{1 \leq i \leq m} h_{i j}\right|}{4} .
\end{aligned}
$$

Since all alternatives are non inferior, we build the following model for the optimal $\lambda$-fuzzy measure on attribute set $\mathrm{C}$.

$$
\begin{gathered}
\min \sum_{\mathrm{i}=1}^{m} \sum_{\mathrm{j}=1}^{\mathrm{n}} \mathrm{d}_{\mathrm{ij}} g_{\lambda^{\prime}}\left(\mathrm{c}_{\mathrm{j}}\right) \\
\text { s.t. }\left\{\begin{array}{l}
g_{\lambda^{\prime}}(\mathrm{C})=1 \\
g_{\lambda^{\prime}}(\mathrm{S}) \leq \mathrm{g}_{\lambda^{\prime}}(\mathrm{T}) \quad \forall \mathrm{S}, \mathrm{T} \subseteq \mathrm{C} \text { s.t.S } \subseteq \mathrm{T}, \\
g_{\lambda^{\prime}}\left(\mathrm{C}_{\mathrm{j}}\right) \in \mathrm{W}_{\mathrm{c}_{\mathrm{j}}}, \mathrm{g}_{\lambda^{\prime}}\left(\mathrm{C}_{\mathrm{j}}\right) \geq 0, \forall \mathrm{C}_{\mathrm{j}} \in \mathrm{C}
\end{array}\right.
\end{gathered}
$$

which is equivalent to the following model

$$
\begin{gathered}
\min \sum_{\mathrm{i}=1}^{m} \sum_{\mathrm{j}=1}^{\mathrm{n}} \mathrm{d}_{\mathrm{ij}} \mathrm{g}_{\lambda^{\prime}}\left(\mathrm{c}_{\mathrm{j}}\right) \\
\text { s.t. } g_{\lambda^{\prime}}\left(c_{\mathrm{j}}\right) \in \mathrm{W}_{\mathrm{c}_{\mathrm{j}}}, \mathrm{g}_{\lambda^{\prime}}\left(\mathrm{c}_{\mathrm{j}}\right) \geq 0, \quad \mathrm{j}=1,2, \ldots, \mathrm{n},
\end{gathered}
$$

where $g_{\lambda^{\prime}}\left(c_{j}\right)$ is the $\lambda$-fuzzy measure of the attribute $c_{j}$, and $W_{c_{j}}$ is the range of the attribute $c_{j}$.

Solve the model (16), we get the optimal $\lambda$-fuzzy measure of each element in C. By Eq.(14), we get the $\lambda$-fuzzy measure of each subset in $C$.

For each $j=1,2, \ldots, n$, calculate

$$
\begin{array}{r}
\mathrm{d}\left(\tilde{r}_{i j}, \tilde{z}_{j}\right)=\left|e_{i j}-\sum_{\mathrm{i}=1}^{\mathrm{m}} \mathrm{e}_{\mathrm{ij}} / \mathrm{m}\right|+\left|\mathrm{f}_{\mathrm{ij}}-\sum_{\mathrm{i=1}}^{\mathrm{m}} \mathrm{f}_{\mathrm{ij}} / \mathrm{m}\right|+ \\
\left|\mathrm{g}_{\mathrm{ij}}-\sum_{\mathrm{i}=1}^{\mathrm{m}} \mathrm{g}_{\mathrm{ij}} / \mathrm{m}\right|+\left|\mathrm{h}_{\mathrm{ij}}-\sum_{\mathrm{i}=1}^{\mathrm{m}} \mathrm{h}_{\mathrm{ij}} / \mathrm{m}\right|
\end{array}
$$

for all $\mathrm{i}=1,2, \ldots, \mathrm{m}$, where

$$
\tilde{z}_{j}=\left(\left[\frac{\sum_{i=1}^{m} e_{i j}}{m}, \frac{\sum_{i=1}^{m} f_{i j}}{m}\right],\left[\frac{\sum_{i=1}^{m} g_{i j}}{m}, \frac{\sum_{i=1}^{m} h_{i j}}{m}\right]\right) .
$$

Reorder $\mathrm{d}\left(\tilde{\boldsymbol{r}}_{i j}, \tilde{z}_{j}\right)$ in ascending order for each $\mathrm{i}=1,2, \ldots, \mathrm{m}$, we $\operatorname{get} d\left(\overline{\tilde{r}}_{i(1)}, \bar{z}_{i}\right) \leq d\left(\overline{\bar{r}}_{i(2)}, \overline{\bar{z}}_{i}\right) \leq \ldots \leq d\left(\overline{\bar{r}}_{i(n)}, \overline{\tilde{z}}_{i}\right)$, where $(\cdot)$ is a permutation on $\mathrm{N}$.

Based on the mean deviation method, we give the following model for the optimal $\lambda$-fuzzy measure on ordered set $N=\{1,2, \ldots, n\}$.

$$
\begin{gathered}
\max \sum_{\mathrm{i}=1}^{\mathrm{m}} \sum_{\mathrm{j}=1}^{\mathrm{n}} \mathrm{d}\left(\tilde{r}_{i(j)}, \tilde{z}_{i}\right) g_{\lambda}(j) \\
\text { s.t. }\left\{\begin{array}{l}
\mathrm{g}_{\lambda}(\mathrm{N})=1 \\
\mathrm{~g}_{\lambda} \$ \Varangle \mathrm{g}_{\lambda} \mathrm{T}(\forall \mathrm{S} \mathrm{T} \subseteq \mathrm{N} \text { st } \mathrm{S} \subseteq \mathrm{T} \\
\mathrm{g}_{\lambda}(\mathrm{j}) \in \mathrm{W}_{\mathrm{j}} \mathrm{g}_{\lambda} \mathrm{j}(\geq) \quad \forall \mathrm{j}, \in \mathrm{N}
\end{array}\right.
\end{gathered}
$$

which is equivalent to the following model

$$
\begin{gathered}
\quad \max \sum_{\mathrm{i}=1}^{\mathrm{m}} \sum_{\mathrm{j}=1}^{\mathrm{n}} \mathrm{d}\left(\tilde{r}_{i(j)}, \tilde{z}_{i}\right) g_{\lambda}(j) \\
\text { s.t. } \mathrm{g}_{\lambda}(\mathrm{j}) \in \mathrm{W}_{\mathrm{j}}, \mathrm{g}_{\lambda}(\mathrm{j}) \geq 0, \quad \mathrm{j}=1,2, \ldots, \mathrm{n},
\end{gathered}
$$

where $g_{\lambda}(j)$ is the fuzzy measure of the $j$ th index, and $W_{j}$ is the range of the $j$ th position.

Solve the model (18), we get the optimal $\lambda$-fuzzy measure of each element in N. By Eq.(14), we get the $\lambda$-fuzzy measure of each subset in $N$.

\subsection{A new method to multi-attribute decision making}

Based on above analysis, we develop an approach to multi-attribute decision making under Atanassov's intervalvalued intuitionistic fuzzy environment. The main decision procedure can be described as follows:

Step 1: If all attributes $c_{j}(j=1,2, \ldots, n)$ are benefits (i.e., the larger, the greater preference), then the attribute values do not need normalization. Otherwise, we normalize the Atanassov's interval-valued intuitionistic fuzzy decision matrix $\tilde{A}=\left(\tilde{\alpha}_{i j}\right)_{m \times n}$ into $\tilde{\mathrm{R}}=\left(\tilde{r}_{i j}\right)_{m \times n}$, where $\tilde{r}_{i j}=\left\{\begin{array}{l}\tilde{\alpha}_{i j} \text { for benefit attribute } \mathrm{c}_{\mathrm{j}} \\ \left(\tilde{\alpha}_{i j}\right)^{C} \text { for cost attribute } \mathrm{c}_{\mathrm{j}}\end{array}\right.$ with $\tilde{\alpha}_{i j}=\left(\left[c_{i j}, d_{i j}\right],\left[a_{\mathrm{ij}}, b_{\mathrm{ij}}\right]\right)(\mathrm{i}=1,2, \ldots, \mathrm{m} ; \mathrm{j}=1,2, \ldots, \mathrm{n})$ 
(see $[53,54])$

Step 2: Solve the model (16), we get the optimal $\lambda$-fuzzy measure on attribute set $C$, and calculate the attribute Shapley values.

Step 3: Solve the model (18), we obtain the optimal $\lambda$-fuzzy measure on ordered set $\mathrm{N}$.

Step 4: According to order-inducing variables $u_{i}(i=1,2, \ldots, n)$, calculate the generalized Shapley values.

Step 5: Apply the IG $\lambda \mathrm{S}$-IVIFHCAA operator

$\tilde{r}_{i}=\mathrm{IG \lambda S} \mathrm{IVIFHCAA}{ }_{\Phi, \varphi}\left(<u_{1}, \tilde{r}_{i 1}>,<u_{2}, \tilde{r}_{i 2}>, \ldots,<u_{n}, \tilde{r}_{i n}>\right)$

$$
=\frac{\bigoplus_{j=1}^{n}\left(\Phi_{A_{(j)}}\left(g_{\lambda}, N\right)-\Phi_{A_{(j+1)}}\left(g_{\lambda}, N\right)\right) \varphi_{c_{(j)}}\left(g_{\lambda^{\prime}}, C\right) \tilde{r}_{i(j)}}{\sum_{j=1}^{n}\left(\Phi_{A_{(j)}}\left(g_{\lambda}, N\right)-\Phi_{A_{(j+1)}}\left(g_{\lambda}, N\right)\right) \varphi_{c_{(j)}}\left(g_{\lambda^{\prime}}, C\right)},
$$

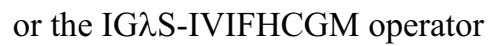

$$
\tilde{r}_{i}=\operatorname{IG} \lambda \mathrm{S}-\mathrm{IVIFHCGM} \mathrm{I}_{, \varphi}\left(<u_{1}, \tilde{r}_{i 1}>,<u_{2}, \tilde{r}_{i 2}>, \ldots,<u_{n}, \tilde{r}_{i n}>\right)
$$$$
=\bigotimes_{\mathrm{j}=1}^{n} \tilde{r}_{i(j)} \frac{\left(\Phi_{A_{(j)}}\left(g_{\lambda}, \mathrm{N}\right)-\Phi_{A_{(j+1)}}\left(g_{\lambda}, \mathrm{N}\right)\right) \varphi_{C_{(j)}}\left(g_{\lambda^{\prime}}, \mathrm{C}\right)}{\sum_{j=1}^{n}\left(\Phi_{A_{(j)}}\left(g_{\lambda}, \mathrm{N}\right)-\Phi_{A_{(j+1)}}\left(g_{\lambda}, \mathrm{N}\right)\right) \varphi_{c_{(j)}}\left(\mathrm{g}_{\lambda^{\prime}}, \mathrm{C}\right)}
$$

to get the comprehensive attribute values

$$
\check{r}_{i}=\left(\left[u_{i}, v_{i}\right],\left[\mathrm{x}_{\mathrm{i}}, \mathrm{y}_{\mathrm{i}}\right]\right) \quad \mathrm{i}=1,2, \ldots, \mathrm{m} .
$$

Step 6: According to the comprehensive attribute value $\tilde{r}_{i}=\left(\left[u_{i}, v_{i}\right],\left[\mathrm{x}_{\mathrm{i}}, \mathrm{y}_{\mathrm{i}}\right]\right)$ of the alternative $\mathrm{a}_{\mathrm{i}}$, calculate the score

$$
S\left(\tilde{r}_{i}\right)=\frac{u_{i}+v_{i}-x_{i}-y_{i}}{2}
$$

and the accuracy degree

$$
H\left(\tilde{r}_{i}\right)=\frac{u_{i}+v_{i}+x_{i}+y_{i}}{2}
$$

Step 7: End.

to rank the alternative $a_{i}(i=1,2, \ldots, m)$, then to select the best one.

\section{A practical numerical example}

Suppose there is an investment company, which wants to invest a sum of money in the best option (adapted from Ref. [55]). There is a panel with four possible alternatives to invest the money: (1) $a_{1}$ is a car company; (2) $a_{2}$ is a food company; (3) $a_{3}$ is a computer company; (4) $a_{4}$ is an arms company. The investment company must take a decision according to the following three attributes: (1) $C_{1}$ is the risk analysis; (2) $C_{2}$ is the growth analysis; (3) $C_{3}$ is the environmental impact analysis. The four possible alternatives are to be evaluated using the Atanassov's interval-valued intuitionistic fuzzy information by the expert under the above three criteria, as listed in the following decision matrix

$\bar{A}=\left(\begin{array}{lll}([0.4,0.5],[0.3,0.4]) & ([0.4,0.6],[0.2,0.4]) & ([0.1,0.3],[0.5,0.6]) \\ ([0.6,0.7],[0.2,0.3]) & ([0.6,0.7],[0.2,0.3]) & ([0.4,0.7],[0.1,0.2]) \\ ([0.3,0.6],[0.3,0.4]) & ([0.5,0.6],[0.3,0.4]) & ([0.5,0.6],[0.1,0.3]) \\ ([0.7,0.8],[0.1,0.2]) & ([0.6,0.7],[0.1,0.3]) & ([0.3,0.4],[0.1,0.2])\end{array}\right)$

Each element of this matrix is an IVIFS, representing the expert's assessment as to what degree an alternative is and is not an excellent investment as per an attribute. For instance, the top-left cell, $([0.4,0.5],[0.3,0.4])$, reflects the expert's belief that alternative $a_{1}$ is an excellent investment from a risk perspective $\left(C_{1}\right)$ with a margin of $40-50 \%$ and $a_{1}$ is not an excellent choice given its risk profile $\left(C_{1}\right)$ with a chance between $30 \%$ and $40 \%$. Assume that the importance of each attribute is defined by $W_{c_{1}}=[0.25,0.4], W_{c_{2}}=[0.35,0.45]$ and $W_{c_{3}}=[0.3,0.5]$, and the importance of each ordered position is given as $W_{1}=[0.2,0.3], W_{2}=[0.4,0.6]$ and $W_{3}=[0.2,0.3]$. In the following, we can utilize the proposed procedure to get the most desirable investment company(s).

Step 1: Since $C_{2}$ (the growth analysis) is benefit attribute, and $C_{1}$ (the risk analysis) and $C_{3}$ (the environmental impact analysis) are cost attributes, we need to normalize the given matrix, as listed in the following.

$\tilde{R}=\left(\begin{array}{lll}([0.3,0.4],[0.4,0.5]) & ([0.4,0.6],[0.2,0.4]) & ([0.5,0.6],[0.1,0.3]) \\ ([0.2,0.3],[0.6,0.7]) & ([0.6,0.7],[0.2,0.3]) & ([0.1,0.2],[0.4,0.7]) \\ ([0.3,0.4],[0.3,0.6]) & ([0.5,0.6],[0.3,0.4]) & ([0.1,0.3],[0.5,0.6]) \\ ([0.1,0.2],[0.7,0.8]) & ([0.6,0.7],[0.1,0.3]) & ([0.1,0.2],[0.3,0.4])\end{array}\right)$.

Step 2: From $\tilde{R}$, we get

$$
\begin{aligned}
\tilde{R}^{+}= & (([0.3,0.4],[0.3,0.5]),([0.6,0.7],[0.1,0.3]), \\
& ([0.5,0.6],[0.1,0.3])), \\
\tilde{R}^{-}= & (([0.1,0.2],[0.7,0.8]),([0.4,0.6],[0.3,0.4]), \\
& ([0.1,0.2],[0.5,0.7])),
\end{aligned}
$$

and the distance matrices $D^{+}=\left(d_{i j}^{+}\right)_{4 \times 3}$ and $D^{-}=\left(d_{i j}^{-}\right)_{4 \times 3}$ as follows: 
F anyong M eng et al.

$$
\mathrm{D}^{+}=\left(\begin{array}{ccc}
0.025 & 0.125 & 0 \\
0.175 & 0.025 & 0.375 \\
0.025 & 0.125 & 0.35 \\
0.375 & 0 & 0.275
\end{array}\right), \mathrm{D}^{-}=\left(\begin{array}{ccc}
0.35 & 0.025 & 0.4 \\
0.1 & 0.125 & 0.025 \\
0.25 & 0.025 & 0.05 \\
0 & 0.15 & 0.125
\end{array}\right) .
$$

From the distance matrices $\mathrm{D}^{+}$and $\mathrm{D}^{-}$, we obtain the following relative distance matrix

$$
\mathrm{D}=\left(\begin{array}{ccc}
0.067 & 0.833 & 0 \\
0.636 & 0.167 & 0.938 \\
0.091 & 0.833 & 0.875 \\
1 & 0 & 0.688
\end{array}\right)
$$

According to the model (16), we get the following model

$$
\min 1.8 \mathrm{~g}_{\lambda^{\prime}}\left(\mathrm{c}_{1}\right)+1.83 \mathrm{~g}_{\lambda^{\prime}}\left(\mathrm{c}_{2}\right)+2.5 \mathrm{~g}_{\lambda^{\prime}}\left(\mathrm{c}_{3}\right)
$$

s.t. $g_{\lambda^{\prime}}\left(c_{1}\right) \in[0.25,0.4], g_{\lambda^{\prime}}\left(c_{2}\right) \in[0.35,0.45], g_{\lambda^{\prime}}\left(c_{3}\right) \in[0.3,0.5]$.

Solve the above linear programming, we obtain

$$
\mathrm{g}_{\lambda^{\prime}}\left(\mathrm{c}_{1}\right)=0.25, \mathrm{~g}_{\lambda^{\prime}}\left(\mathrm{c}_{2}\right)=0.35, \mathrm{~g}_{\lambda^{\prime}}\left(\mathrm{c}_{3}\right)=0.3 \text {. }
$$

By $\prod_{c_{i} \in C}\left[1+\lambda^{\prime} g_{\lambda^{\prime}}\left(C_{i}\right)\right]=1+\lambda^{\prime}$, we get $\lambda^{\prime}=0.361$. From Eq.(14), we obtain

$$
\begin{aligned}
& g_{\lambda^{\prime}}\left(C_{1}, C_{2}\right)=0.63, g_{\lambda^{\prime}}\left(C_{1}, C_{3}\right)=0.58, g_{\lambda^{\prime}}\left(C_{2}, C_{3}\right)=0.69, \\
& g_{\lambda^{\prime}}\left(C_{1}, C_{2}, C_{3}\right)=1 .
\end{aligned}
$$

From Eq.(3), we get the attribute Shapley values

$\varphi_{c_{1}}\left(g_{\lambda^{\prime}}, \mathrm{C}\right)=0.28, \varphi_{c_{2}}\left(g_{\lambda^{\prime}}, \mathrm{C}\right)=0.385, \varphi_{c_{3}}\left(g_{\lambda^{\prime}}, \mathrm{C}\right)=0.335$.

Step 3: By Eq.(17), we get the distance matrix $\mathrm{D}^{\prime}=\left(\mathrm{d}\left(\tilde{r}_{i j}, \tilde{z}_{j}\right)\right)_{4 \times 3}$ as follows:

$$
D^{\prime}=\left(\begin{array}{ccc}
0.4 & 0.225 & 1 \\
0.2 & 0.175 & 0.5 \\
0.4 & 0.225 & 0.4 \\
0.6 & 0.275 & 0.35
\end{array}\right)
$$

According to the model (18), we obtain the following model

$$
\max 0.9 g_{\lambda}(1)+1.35 g_{\lambda}(2)+2.5 g_{\lambda}(3)
$$

s.t. $g_{\lambda}(1) \in[0.2,0.3], g_{\lambda}(2) \in[0.4,0.6], g_{\lambda}(3) \in[0.2,0.3]$.

Solve the above linear programming, we have

$$
g_{\lambda}(1)=0.3, g_{\lambda}(2)=0.6, g_{\lambda}(3)=0.3 \text {. }
$$

By $\prod_{i \in N}\left[1+\lambda g_{\lambda}(i)\right]=1+\lambda$, we get $\lambda=-0.471$. From Eq.(14), we obtain

$g_{\lambda}(1,2)=0.815, g_{\lambda}(1,3)=0.558, g_{\lambda}(2,3)=0.815, g_{\lambda}(1,2,3)=1$.

Step 4: When $u_{j}=d\left(\tilde{r}_{i j}, \tilde{z}_{j}\right)(j=1,2,3)$ for each $\mathrm{i}=1,2,3,4$, from
Eq.(2), we get

$$
\begin{aligned}
& \Phi_{\mathrm{N}}\left(\mathrm{g}_{\lambda}, \mathrm{N}\right)=1, \Phi_{\{1,2\}}\left(\mathrm{g}_{\lambda}, \mathrm{N}\right)=\Phi_{\{2,3\}}\left(\mathrm{g}_{\lambda}, \mathrm{N}\right)=0.758, \\
& \Phi_{1}\left(\mathrm{~g}_{\lambda}, \mathrm{N}\right)=\Phi_{3}\left(\mathrm{~g}_{\lambda}, \mathrm{N}\right)=0.241, \Phi_{2}\left(\mathrm{~g}_{\lambda}, \mathrm{N}\right)=0.519 .
\end{aligned}
$$

Step 5: By the IG $\lambda$ S-IVIFHCAA operator, calculate the comprehensive attribute values $\tilde{r}_{i}(\mathrm{i}=1,2,3,4)$, e.g., $\mathrm{i}=1$,

$$
\begin{aligned}
\tilde{r}_{1}= & \text { IG } \text { S-IVIFHCAA }{ }_{\Phi, \varphi}\left(<u_{1}, \tilde{r}_{11}>,<u_{2}, \tilde{r}_{12}>,<u_{3}, \tilde{r}_{13}>\right) \\
= & \frac{\left(\Phi_{\mathrm{N}}\left(g_{\lambda}, \mathrm{N}\right)-\Phi_{\{1,2\}}\left(g_{\lambda}, \mathrm{N}\right)\right) \varphi_{\mathrm{c}_{3}}\left(\mathrm{~g}_{\lambda^{\prime}}, \mathrm{C}\right) \tilde{r}_{13}}{\left(\Phi_{\mathrm{N}}\left(\mathrm{g}_{\lambda}, \mathrm{N}\right)-\Phi_{\{1,2\}}\left(\mathrm{g}_{\lambda}, \mathrm{N}\right)\right) \varphi_{\mathrm{c}_{3}}\left(\mathrm{~g}_{\lambda^{\prime}}, \mathrm{C}\right)} \oplus \\
& \frac{\left(\Phi_{\{1,2\}}\left(\mathrm{g}_{\lambda}, \mathrm{N}\right)-\Phi_{1}\left(\mathrm{~g}_{\lambda}, \mathrm{N}\right)\right) \varphi_{\mathrm{c}_{2}}\left(\mathrm{~g}_{\lambda^{\prime}}, \mathrm{C}\right) \tilde{r}_{12}}{+\left(\Phi_{\{1,2\}}\left(\mathrm{g}_{\lambda}, \mathrm{N}\right)-\Phi_{1}\left(\mathrm{~g}_{\lambda}, \mathrm{N}\right)\right) \varphi_{\mathrm{c}_{2}}\left(\mathrm{~g}_{\lambda^{\prime}}, \mathrm{C}\right)} \oplus \\
& \frac{\left(\Phi_{1}\left(\mathrm{~g}_{\lambda}, \mathrm{N}\right)-\Phi_{\varnothing}\left(\mathrm{g}_{\lambda}, \mathrm{N}\right)\right) \varphi_{\mathrm{c}_{1}}\left(\mathrm{~g}_{\lambda^{\prime}}, \mathrm{C}\right) \tilde{r}_{11}}{+\left(\Phi_{1}\left(\mathrm{~g}_{\lambda}, \mathrm{N}\right)-\Phi_{\varnothing}\left(\mathrm{g}_{\lambda}, \mathrm{N}\right)\right) \varphi_{\mathrm{c}_{1}}\left(\mathrm{~g}_{\lambda^{\prime}}, \mathrm{C}\right)} \\
= & ([0.41,0.57],[0.19,0.39]) .
\end{aligned}
$$

Similar to the calculation of $\tilde{r}_{1}$, we get the comprehensive attribute values $\tilde{r}_{i}(i=2,3,4)$ as follows:

$$
\begin{aligned}
& \tilde{r}_{2}=([0.45,0.56],[0.29,0.43]), \tilde{r}_{3}=([0.39,0.51],[0.34,0.48]), \\
& \tilde{r}_{4}=([0.44,0.54],[0.19,0.39]) .
\end{aligned}
$$

If the IG $\lambda \mathrm{S}$-IVIFHCGM operator is used in this example, then the comprehensive attribute values $\tilde{r}_{i}^{\prime}(i=1,2,3,4)$ are obtained as follows:

$$
\begin{aligned}
& \tilde{r}_{1}^{\prime}=([0.4,0.55],[0.22,0.4]), \hat{r}_{2}^{\prime}=([0.32,0.44],[0.35,0.51]), \\
& \tilde{r}_{3}^{\prime}=([0.31,0.47],[0.35,0.5]), \hat{r}_{4}^{\prime}=([0.28,0.41],[0.31,0.47]) .
\end{aligned}
$$

Step 5: According to the comprehensive attribute values $\tilde{r}_{i}$ $(i=1,2,3,4)$, we get the following scores

$$
\mathrm{S}\left(\tilde{r}_{1}\right)=0.2, \mathrm{~S}\left(\tilde{r}_{2}\right)=0.145, \mathrm{~S}\left(\tilde{r}_{3}\right)=0.04, \mathrm{~S}\left(\tilde{r}_{4}\right)=0.2 \text {, }
$$

and the following accuracy degrees

$$
\mathrm{H}\left(\tilde{r}_{1}\right)=0.78, \mathrm{H}\left(\tilde{r}_{2}\right)=0.865, \mathrm{H}\left(\tilde{r}_{3}\right)=0.86, \mathrm{H}\left(\tilde{r}_{4}\right)=0.78 \text {. }
$$

From the comprehensive attribute values $\tilde{r}_{i}^{\prime}(i=1,2,3,4)$, we obtain the following scores

$$
\begin{gathered}
\mathrm{S}\left(\tilde{r}_{1}^{\prime}\right)=0.165, \mathrm{~S}\left(\tilde{r}_{2}{ }^{\prime}\right)=-0.05, \\
\mathrm{~S}\left(\tilde{\widetilde{r}}_{3}{ }^{\prime}\right)=-0.035, \mathrm{~S}\left(\tilde{\boldsymbol{r}}_{4}{ }^{\prime}\right)=-0.045 .
\end{gathered}
$$

Step 6: From $S\left(\tilde{r}_{i}\right)$ and $H\left(\tilde{r}_{i}\right)(i=1,2,3,4)$, we get

$$
\tilde{r}_{1}=\tilde{r}_{4}>\tilde{r}_{2}>\tilde{r}_{3} \text {. }
$$

By $S\left(\tilde{r}_{i}^{\prime}\right)(i=1,2,3,4)$, we obtain $\tilde{r}_{1}>\tilde{r}_{2}>\tilde{r}_{3}>\tilde{r}_{4}$.

Thus, the car company $a_{1}$ is the best choice. 
Based on the Choquet integral, $\mathrm{Xu}$ [31] defined the following interval-valued intuitionistic fuzzy correlated averaging (IVIFCA) operator

$$
\operatorname{IVIFCA}\left(\ddot{\alpha}_{1}, \tilde{\alpha}_{2}, \ldots, \tilde{\alpha}_{n}\right)=\bigoplus_{i=1}^{\grave{n}} \tilde{\alpha}_{(i)}\left(\mu\left(A_{(i)}\right)-\mu\left(\mathrm{A}_{(i+1)}\right)\right),
$$

and $\mathrm{Xu}$ [31] and Tan [33] respectively presented the following interval-valued intuitionistic fuzzy geometric mean (IVIFGM) operator

$$
\operatorname{IVIFGA}\left(\tilde{\alpha}_{1}, \tilde{\alpha}_{2}, \ldots, \tilde{\alpha}_{n}\right)=\bigotimes_{\mathrm{i}=1}^{\mathrm{n}} \tilde{\alpha}_{(i)}^{\mu\left(A_{(i)}\right)-\mu\left(\mathrm{A}_{(i+1)}\right)},
$$

where $(\cdot)$ indicates a permutation on $X$ such that $\tilde{\alpha}_{(1)} \leq \tilde{\alpha}_{(2)} \leq$, $\ldots \leq \tilde{\alpha}_{(n)}$ and $\mathrm{A}_{(\mathrm{i})}=\left\{\tilde{\alpha}_{(i)}, \ldots, \tilde{\alpha}_{(n)}\right\}$ with $\mathrm{A}_{(\mathrm{n}+1)}=\varnothing, \mu$ is the fuzzy measure on $\mathrm{A}=\left\{\tilde{\tilde{\alpha}}_{i}\right\}_{i \in N}$.

Based on the Shapley function, Zhang et al. [40] defined the following Shapely value-based intuitionistic fuzzy aggregation (SIFA) operator

$$
\operatorname{SIFA}\left(\tilde{\alpha}_{1}, \tilde{\alpha}_{2}, \ldots, \tilde{\alpha}_{n}\right)=\bigoplus_{i=1}^{\bar{n}} \varphi_{\tilde{\alpha}_{i}}(\mu, A) \tilde{\alpha}_{i},
$$

where $\varphi_{\tilde{\alpha}_{i}}(\mu, A)$ is the Shapley value with respect to the fuzzy measure $\mu$ on $\mathrm{A}=\left\{\tilde{\alpha}_{i}\right\}_{i \in N}$ for IFV $\tilde{\alpha}_{i}(\mathrm{i}=1,2, \ldots, \mathrm{n})$.

Although the IVIFCA and IVIFGM operators can reflect the interactions among elements, they only consider the correlative characteristics between the combinations $A_{(i)}$ and $A_{(i+1)}(i=1,2, \ldots, n)$. As we know, when there exist interactions among elements, it is obviously unsuitable to only consider these two combinations. Furthermore, the SIFA operator neither considers the importance of their ordered positions, nor does it reflect the interactions among them.

For the comparative convenience, the ranking results w.r.t. the different aggregation operators are obtained in Table 1.

Table 1 Ranking orders w.r.t. the different aggregation operators

\begin{tabular}{cl}
\hline Operator & Ranking order \\
\hline The IVIFCA operator & $\tilde{r}_{1}>\tilde{r}_{4}>\tilde{r}_{2}>\tilde{r}_{3}$ \\
The IVIFGM operator & $\tilde{r}_{1}>\tilde{r}_{3}>\tilde{r}_{4}>\tilde{r}_{2}$ \\
The SIFA operator & $\tilde{r}_{1}>\tilde{r}_{4}>\tilde{r}_{2}>\tilde{r}_{3}$ \\
The IG $\lambda$ S-IVIFHCAA operator & $\tilde{r}_{1}=\tilde{r}_{4}>\tilde{r}_{2}>\tilde{r}_{3}$ \\
The IG $\lambda$ S-IVIFHCGM operator & $\tilde{r}_{1}>\tilde{r}_{2}>\tilde{r}_{3}>\tilde{r}_{4}$ \\
\hline
\end{tabular}

From this example, it can be observed that different ranking results are yielded by using different aggregation operators, and the optimal alternatives maybe also different. Thus, decision makers can properly select the aggregation operators according to the underlying interest and demands to each practical problem.

\section{Conclusion}

We have developed two induced hybrid aggregation operators under Atanassov's interval-valued intuitionistic fuzzy environment based on the Choquet integral and the generalized Shapley function, which do not only globally consider the importance of elements and their ordered positions, but also overall reflect the correlations among them and their ordered positions. Since the fuzzy measure is defined on the power set, it makes the problem exponentially complex. Thus, it is not easy to get the fuzzy measure of each combination in a set when it is large. For this reason, we further define the IG $\lambda$ S-IVIFHCAA and IG $\lambda$ SIVIFHCGM operators, which greatly simplify the complexity of solving a fuzzy measure. If the information about the weighting vectors is incompletely known, the models for the optimal $\lambda$-fuzzy measures on attribute set and ordered set are built, respectively. Moreover, an approach to Atanassov's interval-valued intuitionistic fuzzy multi- attribute decision making is developed, and a financial decision making problem under Atanassov's interval-valued intuitionistic fuzzy environment has been provided to illustrate our approach. The numerical results showed that different optimal alternatives may be yielded by using different aggregation operators, and thus, the decision maker can properly select the desirable alternative according to his interest and the actual needs.

\section{A cknowledgment}

The authors first gratefully thank the Area Editor Prof. Humberto Bustince and two anonymous referees for their valuable comments, which have much improved the paper. This work was supported by the National Natural Science Foundation Youth Project of China (No. 71201089), the National Natural Science Foundation of China (Nos. 71071018 and 71271217), the Natural Science Foundation Youth Project of Shandong Province, China (ZR2012GQ005), and the Specialized Research Fund for the 
F anyong M eng et al.

Doctoral Program of Higher Education (No. 20111101110036).

\section{References}

[1] R. R. Yager, On ordered weighted averaging aggregation operators in multicriteria decision making, IEEE Tran. System, M an Cybernet., 18(1) (1988) 183-190.

[2] R. R. Yager, On a semantics for neural networks based on fuzzy quantifiers, Int. J . Intell. Syst., 7(8) (1992) 765-786.

[3] D. P. Filev and R. R. Yager, On the issue of obtaining OWA operator weightings, F uzzy Sets Syst., 94(2) (1998) 157-169.

[4] R. Fullér and P. Majlender, On obtaining minimal variability OWA operator weightings, F uzzy Sets Syst., 124(1) (2001) 53- 57.

[5] T. Calvo, G. Mayor and R. Mesiar, Aggregation operators: New trends and applications (New York, Physica-Verlag, 2002).

[6] G. Beliakov, Learning weightings in the generalized OWA operators, F uzzy 0 ptim. Decis. Ma., 4(2) (2005) 119-130.

[7] B. S. Ahn, The uncertain OWA aggregation with weighting functions having a constant level of orness, Int. J . Intell. Syst., 21(5) (2006) 469-483.

[8] Z. S. Xu, Induced uncertain linguistic OWA operators applied to group decision making, Inform. F usion, 7(2) (2006) $231-238$.

[9] Z. S. Xu, Dependent uncertain ordered weighted aggregation operators, Inform. Fusion, 9(2) (2008) 310-316.

[10] Y. M. Wang, Y. Luo and X. W. Liu, Two new models for determining OWA operator weightings, Comput. Ind. Eng., 52(2) (2007) 203-209.

[11] Y. M. Wang and C. Parkan, A preemptive goal programming method for aggregating OWA operator weightings in group decision making, Inform. Sci., 177(8) (2007) 1867-1877.

[12] A. Emrouznejad and G. R Amin, Improving minimax disparity model to determine the OWA operator weightings, Inform. Sci., 180(8) (2010) 1477-1485.

[13] J. M. Merigo and A. M. Gil-Lafuente, The induced generalized OWA operator, Inform. Sci., 179(6) (2009) 729741.

[14] Y. J. Xu and H. M. Wang, The induced generalized aggregation operators for intuitionistic fuzzy sets and their application in group decision making, Appl. Soft Comput., 12(3) (2012) 1168-1179.

[15] Z. S. Xu and R. R. Yager, Some geometric aggregation operators based on intuitionistic fuzzy sets, Int. J. Gen. Syst, 35(4) (2006) 417-433.

[16] R. R. Yager and Z. S. Xu, The continuous ordered weighted geometric operator and its application to decision making, F uzzy Sets Syst., 157(10) (2006) 1393-1402.

[17] J. Wu, J. C. Li, H. Li and W. Q. Duan, The induced continuous ordered weighted geometric operators and their application in group decision making, Comput. Ind. Eng., 56(4) (2009) 1545-1552.

[18] J. Wu, Q. W. Cao and J. L. Zhang, An ILOWG operator based group decision making method and its application to evaluate the supplier criteria, $M$ ath. Comput. M odel., 54(1-2) (2011) 19-34.

[19] G. W. Wei, Some induced geometric aggregation operators with intuitionistic fuzzy information and their application to group decision making, Appl. Soft Comput., 10(2) (2010) 423-431.

[20] Z. S. Xu and J. Chen, On geometric aggregation over interval-valued intuitionistic fuzzy information, Proc. Int. Conf. F uzzy Sys. Knowl. D iscov. F SK D, Haikou, China, 2007 , pp. 466-471.

[21] Z. S. Xu and J. Chen, Approach to group decision making based on interval valued-intuitionistic judgment matrices, Systems Engineering-Theory and Practice, 27(1) (2007) 126-133.

[22] Z. S. Xu and Q. L. Da, An overview of operators for aggregating information, Int. J. Intell. Syst., 18(9) (2003) 953-969.

[23] J. Lin and Y. Jiang, Some hybrid weighted averaging operators and their application to decision making, Inform. F usion http://dx.doi.org/10.1016/j.inffus.2011.06.001.

[24] J. M. Merigo and M. Casanovas, The induced generalized hybrid averaging operator and its application in financial decision making, Int. J. Bus. E con. Finance Manag. Sci., 2(1) (2009) 95- 101.

[25] Z. S. Xu, A method based on linguistic aggregation operators for group decision making with linguistic preference relations, Inform. Sci., 166(1) (2004) 19-30.

[26] Z. S. Xu, Uncertain linguistic aggregation operators based approach to multiple attribute group decision making under uncertain linguistic environment, Inform. Sci., 168(1-4) (2004) 171-184.

[27] H. Zhao, Z. S. Xu, M. F. Ni and S. H. Liu, Generalized aggregation operators for intuitionistic fuzzy sets, Int. J. Intell. Syst., 25(1) (2010) 1-30.

[28] M. Grabisch, Fuzzy integral in multicriteria decision making, F uzzy Sets Syst., 69(3) (1995) 279-298.

[29] M. Grabisch, The application of fuzzy integrals in multicriteria decision making, Eur. J . O per. Res. 89(3) (1996) 445-456.

[30] R. R. Yager, Induced aggregation operators, F uzzy Sets Syst., 137(1) (2003) 59-69.

[31] Z. S. Xu, Choquet integrals of weighted intuitionistic fuzzy information, Inform. Sci., 180(5) (2010) 726-736.

[32] C. Q. Tan and X. H. Chen, Intuitionistic fuzzy Choquet integral operator for multi-criteria decision making, Expert Syst. Appl., 37(1) (2010) 149-157. 
[33] C. Q. Tan, A multi-criteria interval-valued intuitionistic fuzzy group decision making with Choquet integral-based TOPSIS, Expert Syst. Appl., 38(4) (2011) 3023-3033.

[34] C. Q. Tan and X. H. Chen, Induced intuitionistic fuzzy Choquet integral operator for multi-criteria decision making, Int. J . Intell. Syst., 26(7) (2011) 659-686.

[35] L. G. Zhou and H. Y. Chen, Continuous generalized OWA operator and its application to decision making, Fuzzy Sets Syst., 168(1) (2011) 18-34.

[36] Z. S. Xu and M. M. Xia, Induced generalized intuitionistic fuzzy operators, Knowl-Based Syst., 24(2) (2011) 197-209.

[37] C. Q. Tan and X. H. Chen, Induced Choquet ordered averaging operator and its application to group decision making, Int. J . Intell. Syst., 25(1) (2010) 59-82.

[38] C. Q. Tan, D. S. D. Wu and B. J. Ma, Group decision making with linguistic preference relations with application to supplier selection, Expert Syst. Appl., 38(12) (2011) 1438214389.

[39] C. Q. Tan, Generalized intuitionistic fuzzy geometric aggregation operator and its application to multi-criteria group decision making, Soft Comput. 15(5) (2011) 867-876.

[40] X. M. Zhang, Z. S. Xu and X. H. Yu, Shapley value and Choquet integral-based operators for aggregating correlated intuitionistic fuzzy information, Information-TOKYO., 14 (6) (2011) 1847-1858.

[41] G. Choquet, Theory of capacities, Ann. I. Fourier, 5 (1953) 131-295.

[42] Z. S. Xu and Q. L. Da, Combined weighted geometric averaging operator and its application, Journal of Southeast Universit., 32(3) (2002) 506-509.

[43] K. Atanassov and G. Gargov, Interval-valued intuitionistic fuzzy sets, F uzzy Sets Syst., 31(3) (1989) 343-349.

[44] Z.S. Xu, Intuitionistic preference relations and their application in group decision making, Inform. Sci.,177(11) (2007) 2363-2379.

[45] K. Atanassov, Operators over interval-valued intuitionistic fuzzy sets, F uzzy Sets Syst., 64(1) (1994) 159-174.

[46] Z. S. Xu, Intuitionistic fuzzy information: aggregation theory and applications, Beijing: Science Press, 2008.

[47] M. Sugeno, Theory of fuzzy integral and its application, Doctorial Dissertation, Tokyo Institute of Technology, 1974.

[48] M. Grabisch, k-order additive discrete fuzzy measures and their representation, F uzzy Sets Syst., 92(2) (1997) 167-189.

[49] P. Miranda, M. Grabisch and P. Gil, p-Symmetric fuzzy measures. Int. J. Uncertain. F uzz., 10(Suppl.)(1) (2002) 105123.

[50] D. Dubois and H. Prade, Possibility Theory: An Approach to Computerized Processing of Uncertainty, Plenum Press, New York, 1988.

[51] J. L. Marichal, The influence of variables on pseudo-Boolean functions with applications to game theory and multicriteria decision making, Discrete Appl. Math. 107(1-3) (2000) 139164.

[52] L. S. Shapley, A value for n-person game, in: H. Kuhn, A. Tucker (Eds.), Contributions to the theory of games, Princeton University Press, Princeton, 1953.

[53] Z. S. Xu and X. Q. Cai, Nonlinear optimization models for multiple attribute group decision making with intuitionistic fuzzy information, Int. J. Intell. Syst. 25(6) (2010) 489-513.

[54] Z. S. Xu and H. Hu, Projection models for intuitionistic fuzzy multiple attribute decision making, Int. J . Inf. Tech. Decis., 9 (2) (2010) 267-280.

[55] J. Ye, Multicriteria fuzzy decision-making method based on a novel accuracy function under interval-valued intuitionistic fuzzy environment, Expert Syst. Appl., 36(3) (2009) 68996902 . 